\title{
Original paper \\ Geological position and origin of augen gneisses from the Polička Unit, eastern Bohemian Massif
}

\author{
David BURIÁNEK*, Kristýna HRDLIČKOVÁ, Pavel HANŽL \\ Czech Geological Survey, Leitnerova 22, Brno, 658 59, Czech Republic; david.burianek@geology.cz \\ * Corresponding author
}

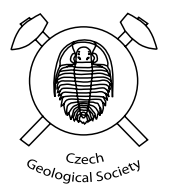

\begin{abstract}
Augen gneisses are a peculiar lithological type of the Polička Unit (PU), which can be divided into two main petrographic groups: augen metagranitoids and migmatitic augen gneisses. Both of the groups represent products of magmatic and metamorphic processes along the contact between the Svratka and Polička units in the eastern Bohemian Massif.

Tonalitic to granodioritic synkinematic intrusions were emplaced into metasedimentary rocks of the Polička Unit during the Late Variscan orogeny, forming plutonic bodies and dykes. Residual fluids from granitic melts pervasively infiltrated the metasedimentary protolith causing a fluid-enhanced partial melting. The P-T conditions of partial melting in the southern part of the PU were estimated at $\sim 750-800{ }^{\circ} \mathrm{C}$ and $7 \mathrm{kbar}$. Melting produced porphyroblastic migmatites with a granite composition. The characteristic augen textures developed from granitic and migmatitic protoliths during a high-temperature deformation in subsolidus conditions. Both the augen metagranitoids and the migmatitic augen gneisses were affected by a subsequent deformation in the shear zone related with thrusting of the Polička Unit over the Svratka Unit.
\end{abstract}

Keywords: structural geology, petrology, geochemistry, geothermobarometry, augen gneiss, Polička Unit Received: 10 March 2009; accepted 29 May 2009; handling editor: M. Štemprok

The online version of this article (http://dx.doi.org/10.3190/jgeosci.043) contains supplementary electronic material.

\section{Introduction}

Augen gneiss is a general term for gneissic rocks containing oval or lenticular crystals or their aggregates usually composed of feldspars. The feldspar porphyroblasts or porphyroclasts vary from $\mathrm{mm}$ to $\mathrm{cm}$ sizes. Augen gneisses are common and widely distributed metamorphic rocks in orogenic belts the genesis of which is variously interpreted. Vassallo and Vernon (2000) explained the augen gneisses as pre- to syn- kinematic intrusions of megacrystic granite. Hutton and Reavy (1992), Vernon and Paterson (2002) and some others described augen gneisses as a product of deformation, especially in shear zones. Augen gneisses were also interpreted as results of metasomatic processes in metasediments. For instance, Pinarelli et al. (2008) explained development of K-feldspar bearing augen gneisses by a model of infiltration of residual hydrous magmas into a metasedimentary protolith. Other types of augen gneisses are thought to be formed by segregation of feldspar (and quartz) crystals and aggregates during migmatization (Mehnert 1968). Generally it is assumed that K-feldspar porphyroblasts are typical of deformed granites, whereas plagioclase forms augen in gneiss of the diorite or tonalite protoliths (Buriánek ed. 2009).

Dark grey gneisses with oval feldspar megacrysts (augen) up to several $\mathrm{mm}$ in size (known also as pearl gneisses in the Central European literature) are conspicu- ous rocks of the Polička Unit (PU) in the eastern Bohemian Massif. Although the works cited above deal mostly with K-feldspar augen in (granitic) gneisses, those from granitic-granodioritic gneisses of the PU have predominantly a plagioclase composition.

Even though the augen gneisses in the PU bear distinctive metamorphic fabrics with penetrative foliation and banding suggesting a metasedimentary protolith, the relics of magmatic fabric are also present. The fuzzy and transitional contacts with surrounding paragneisses also complicate the interpretation of their origin. This fact lead different authors to various genetic interpretations - for example, Melichar ed. (2008) designated them as deformed tonalites and granodiorites, on the other hand Mísař (1961) described them as migmatites.

Not only the apparent contrast between the presence of plagioclase in augen and prevailing granitic to granodioritic bulk chemical composition of the Polička augen gneiss merits discussion. The distinctive textures and homogenous geochemistry of augen gneisses make them a potentially useful lithological marker in a geological reconstruction of $\mathrm{E}$ and NE parts of the Bohemian Massif.

\section{Geological position}

The Polička Unit (PU) is situated in the E part of the Bohemian Massif and in the classic concept of Mísař et 


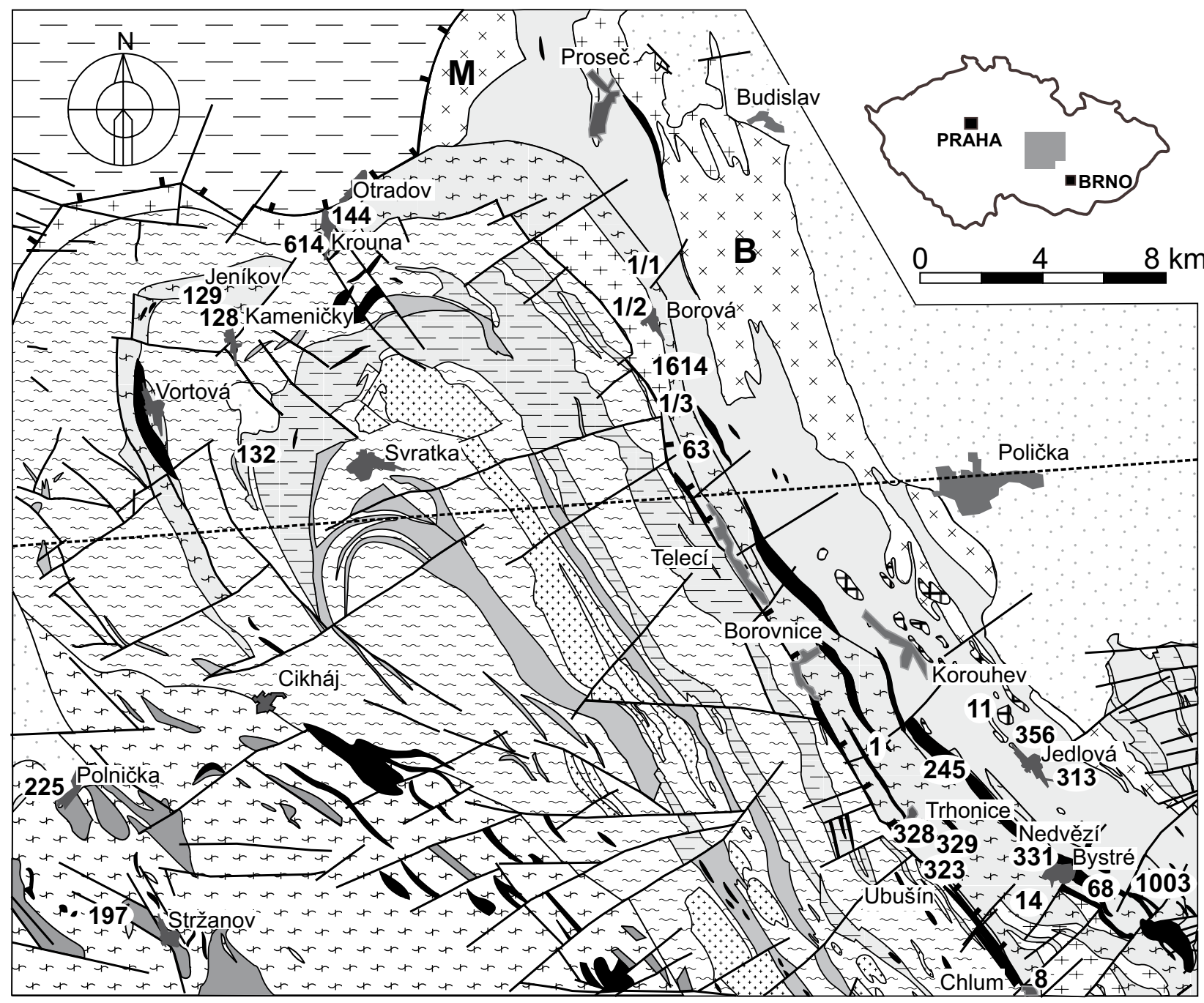

\begin{tabular}{|c|c|}
\hline & Cretaceous sediments \\
\hline & Hlinsko Unit \\
\hline 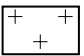 & Granite \\
\hline$x^{x}$ & Granodiorite, tonalite \\
\hline$\rightarrow$ & Gabbro \\
\hline 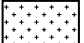 & Metagranite \\
\hline
\end{tabular}

\begin{tabular}{l|l|l} 
Orthogneiss, granulite & \multicolumn{1}{|c}{ Lithological boundary } \\
Augen gneiss & \multicolumn{1}{|c|}{ Fault, normal fault } \\
Migmatite in SU and MZ & $\mathbf{3 2 9}$ & Sample position \\
Paragneiss in PU, SU, MZ & $\mathbf{B}$ & Budislav Pluton \\
Mica schist & $\mathbf{M}$ & Miřetín Pluton \\
Amphibolite, calc silicate & $\ldots$ Cross-section
\end{tabular}

Polička

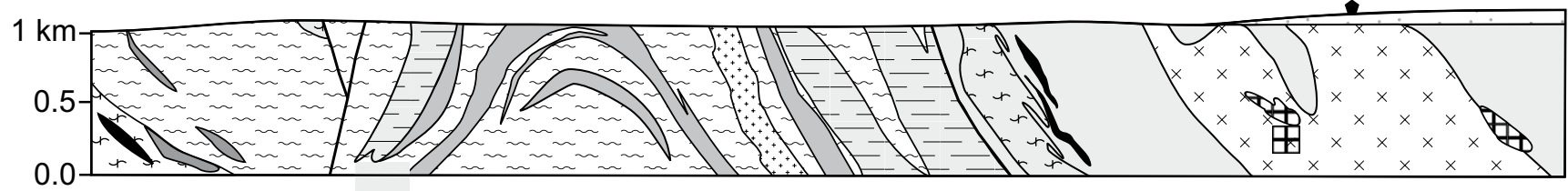

Fig. 1 Geological map of the Svratka and Polička units and of the NE part of Moldanubian Zone at the eastern margin of the Bohemian Massif. Sample locations and W-E geological cross-section are also shown. PU - Polička Unit, SU - Svratka Unit, MZ - Moldanubian Zone. Modified from Melichar ed. (2008), Mrázová ed. (2008), Hanžl ed. (2008), Rejchrt ed. (2009), Buriánek ed. (2009), Čech ed. (2009) and Stárková and Macek (1994). 
al. (1983) it belongs to the Bohemicum. The main part of this unit is exposed in a NW-SE oriented belt (Fig. 1) approximately between Proseč and Bystré. Generally, the PU occurs between the metamorphic rocks of the Svratka Unit in the SW, and the Lower Palaeozoic volcanosedimentary complex of the Hlinsko Unit in the NW. Contact of the PU with the Svratka Unit in the footwall is marked by a narrow discontinuous belt of amphibolites. The boundary was subsequently tectonically reworked by younger, steep NNW-SSE oriented normal faults (Melichar 1993). The Hlinsko Unit in the hanging wall of PU is separated from metamorphic rocks by a significant normal fault of NE-SW to E-W strike. Sediments of the Bohemian Cretaceous basin cover the rocks of PU at the NE.

A belt with equivalents of Polička augen gneisses, newly recognised during the geological survey of the Žd'árské vrchy area (Hanžl ed. 2008; Hrdličková ed. 2008; Mrázová ed. 2008; Buriánek ed. 2009; Čech ed. 2009 and Rejchrt ed. 2009) is exposed as a E-W oriented tectonic slice inside the Svratka Unit between Krouna, Kameničky nad Vortová. There it bends to $\mathrm{S}$ towards Cikháj and continues further to the Moldanubian Zone, where it is exposed in an isolated relic near Stržanov.

The PU is built by three main lithotectonic sequences (Melichar 1993) intruded by several magmatic bodies. The variegated lower subunit forms the base of the PU and it is overlain by a monotonous middle subunit and the topmost metasedimentary upper subunit in the tectonic hanging wall. All subunits are elongated parallel with the regional metamorphic foliation which is NWSE striking and dipping at medium angle towards the NE in the main part of PU between Proseč and Bystré. Foliations in western blocks of the PU follow the strike of the boundary between the Hlinsko and Svratka units (Fig. 2). The strike of the belt with augen gneiss further to SW between Krouna and Cikháj is concordant with foliation in adjacent rocks of the Svratka Unit. This is in agreement with the concept of antiform structure of the unit recognised firstly by Rosiwal (1895).

During the Variscan orogeny, the PU was intruded by a number of intrusive bodies. The following magmatic suites were distinguished by Buriánek et al. (2003) (1) the earliest basic suite of gabbros and diorites, (2) tonalite suite and (3) the latest granite suite.

The most widespread tonalite suite forms the Budislav Pluton which is exposed in a $\mathrm{N}$ part of the PU and is partially covered by Cretaceous sediments. Main elongated body situated between Budislav and Polička is concordant with the NNW-SSE oriented structural trend in this unit and it is accompanied by small satellite bodies towards SE. The Budislav Pluton was dated at $350 \pm 5 \mathrm{Ma}$ by U-Pb method on zircon (Vondrovic and Verner 2008).
Miřetín Pluton is situated in the NW prolongation of the PU and rims the contact between the Polička and Hlinsko units. The P-T conditions of intrusion were estimated at c. $660^{\circ} \mathrm{C}$ and 3-4 kbar (Hanžl ed. 2008). This body is built by deformed rocks of tonalite suite (Buriánek et al. 2003) dated by U-Pb zircon method at $348 \pm 7 \mathrm{Ma}$ (Vondrovic and Verner 2008). Pitra and Guiard (1996) interpreted the Miřetín Pluton as a late Variscan syn-tectonic tonalite intrusion related to a normal ductile shear zone developed along contact between the Svratka and Hlinsko regions.

\subsection{The lower subunit}

The variegated, lower subunit is dominantly exposed along the boundary with the underlying Svratka Unit. It is represented by medium-grained biotite and muscovitebiotite gneisses with strongly deformed bodies of metagranitoids, augen gneisses, amphibolites, marbles and calc-silicate rocks. Intrusive rocks of the Miřetín Pluton intruded the NW part of the lower subunit.

The lower subunit of the PU has been referred to as "tectonic melange" (Buriánek ed. 2009) This is based on a set of geological observations, of which the most convincing seems to be a retrogressive change in metamorphic grade from a granulite-facies in the south of the lower subunit to the amphibolite-facies recorded from the metapelites in its northern part (Buriánek ed. 2009).

The granulites from the lower subunit (Vír granulite body) preserved the P-T conditions of $850-900{ }^{\circ} \mathrm{C}$ and 13-14 kbar (Tajčmanová et al. 2005). Granulite-facies metamorphic mineral association is partly replaced by decompression-related, retrograde mineral assemblage formed at c. $600{ }^{\circ} \mathrm{C}$ and $6-8 \mathrm{kbar}$ (Štoudová et al. 1999). Surrounding metapelites and amphibolites contain evidence for decompression partial melting during uplift. The melt formation within amphibolites was dated at $339.1 \pm 1.1 \mathrm{Ma}$ using single grain zircon evaporation ${ }^{207} \mathrm{~Pb} /{ }^{206} \mathrm{~Pb}$ method (Schulmann et. al 2005).

Augen gneisses are characteristic of the lower subunit of the PU. They form several elongated bodies and layers along the contact with the Svratka Unit having the composition of biotite and amphibole-biotite gneisses. Sillimanite and/or garnet are locally present mainly in the rocks of SE part of the PU. Metamorphic fabric is characterized by penetrative foliation, mineral lineation and banding: non-coaxial deformation is evidenced by asymmetric plagioclase augen. Banding in these rocks is formed by an alternation of leucocratic, quartzplagioclase (minor K-feldspar) bands with darker ones containing variable amounts of biotite and, in the northern part of PU, amphibole. Bodies and layers of augen gneisses are usually enclosed in biotite to muscovitebiotite gneisses or gradually pass from intrusive rocks. 

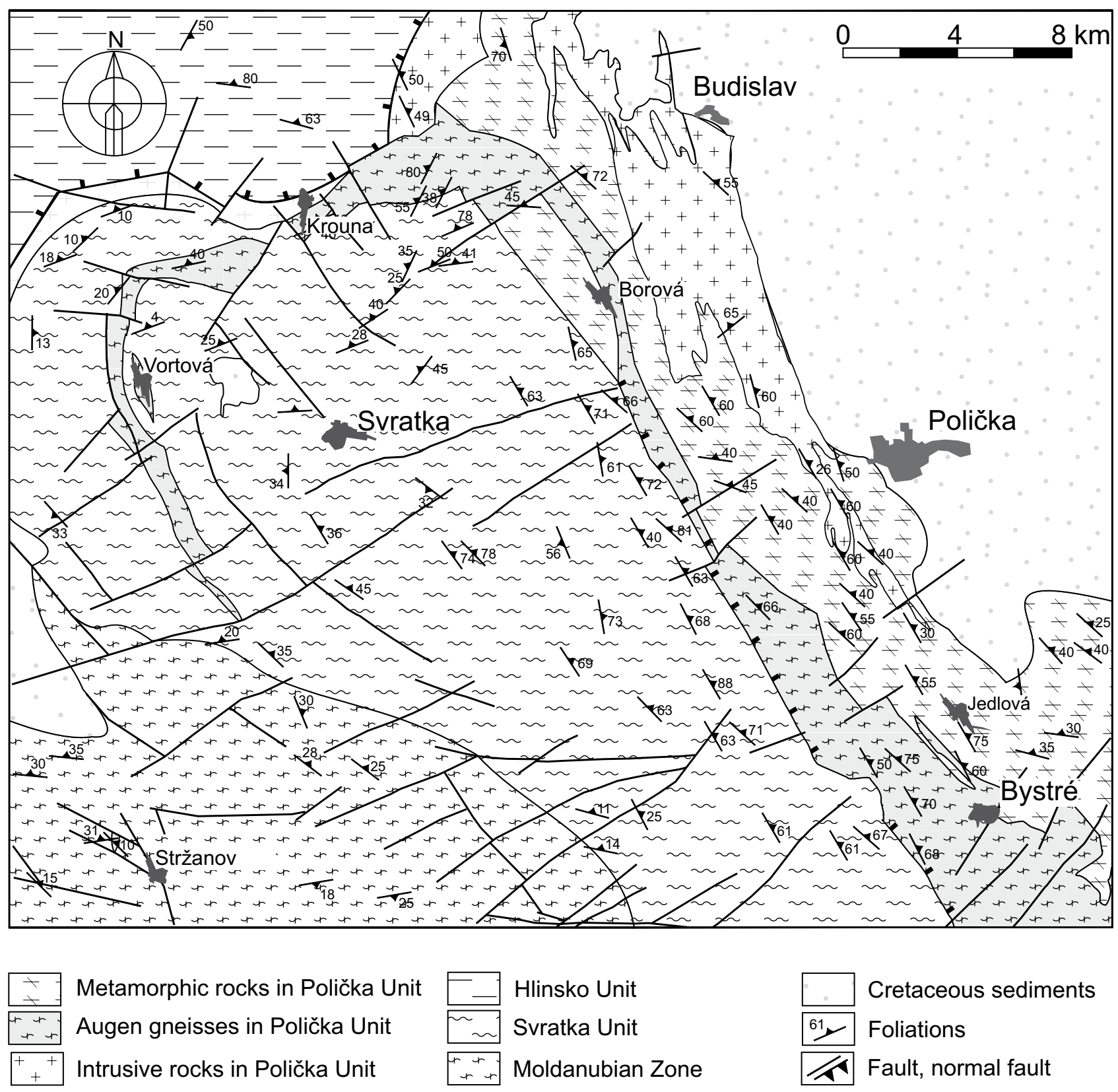

Metamorphic rocks in Polička Unit
Augen gneisses in Polička Unit
Intrusive rocks in Polička Unit

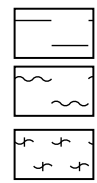

Hlinsko Unit

Svratka Unit

Moldanubian Zone

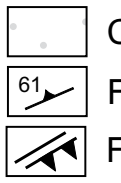

Cretaceous sediments

Foliations

Fault, normal fault

Fig. 2 Simplified tectonic map of the foliations in the Svratka and Polička units and in the NE part of the Moldanubian Zone.

Augen gneisses in elongated bodies along the boundary between the PU and Svratka Unit represent a dominant member in the lowest group of the PU (Fig. 1). The equivalents of the strongly deformed augen gneisses west of the main part of PU were found in tectonic relicts forming several smaller outcrops within leucocratic migmatites of the Svratka Unit between Krouna and Cikháj and within stromatitic migmatites of the Moldanubian Zone near Stržanov. Augen gneisses are associated with deformed porphyritic metadiorites corresponding to the rocks of Miřetín Pluton (Buriánková et al. 2006).

\subsection{The middle subunit}

The middle subunit of the PU is built by monotonous complex of medium-grained paragneisses (metagreywackes, metapelites, rare metaconglomerates) with locally abundant calc-silicate nodules up to $0.5 \mathrm{~m}$ in size. Amphibolites with MORB-like geochemistry form a narrow belt along the boundary of the lower and middle subunits of the PU (Melichar and Hanžl 1997). The second belt of amphibolites rims the contact of the PU with the Svratka Unit (Fig. 1).

Rocks of the middle subunit were intruded by the Budislav Pluton and its equivalents. Submagmatic to 
solid-state deformation fabrics in tonalites are concordant with the structures of the surrounding metamorphic rocks and point to a syn-tectonic character of the intrusion. Amphibole-plagioclase thermometer (Holland and Blundy 1994) together with amphibole barometer (Anderson and Smith 1995) were used for calculation of the P-T conditions in the rocks of the tonalite suite of the Budislav Pluton. The obtained temperatures are between 655 and $735^{\circ} \mathrm{C}$ and pressures between 4 and 6 kbar (Buriánek et al. 2003; Buriánek ed. 2009).

The middle subunit shows an anticlockwise metamorphic path. Relics of the oldest LP-MT metamorphism $\left(\mathrm{M}_{1}\right)$ are preserved mainly in the northernmost part of the unit (Buriánek ed. 2009). Later metamorphism at $580-680{ }^{\circ} \mathrm{C}$ and $5-7 \mathrm{kbar}$ affected a substantial part of the PU $\left(\mathrm{M}_{2}\right)$. This metamorphic event was, in the vicinity of tonalite bodies and in the southern part of the PU, accompanied by a partial melting of the metapelites.

\subsection{The upper subunit}

The upper subunit of PU is represented by a metasedimentary sequence consisting of micaschists with quartzite and paragneiss intercalations. It was found in the eastern exposures of the PU near Stašov, outside the studied area (Melichar 1993).

\section{Methodology}

Mineral analyses were performed on the electron microprobe Cameca SX-100 at the Department of Geological Sciences, Faculty of Science, Masaryk University in Brno. The measurements were carried out in a wave dispersion mode under the following conditions: $15 \mathrm{kV}$ acceleration voltage, $5 \mu \mathrm{m}$ diameter of the electron beam, $30 \mathrm{nA}$ current, integration time 20 seconds, operator $\mathrm{R}$. Čopjaková. The $\mathrm{K}_{\alpha} \mathrm{X}$-ray lines were used as standard: augite $(\mathrm{Si}, \mathrm{Mg})$, orthoclase $(\mathrm{K})$, jadeite $(\mathrm{Na})$, chromite $(\mathrm{Cr})$, almandine $(\mathrm{Al})$, andradite $(\mathrm{Fe}, \mathrm{Ca})$, rhodonite $(\mathrm{Mn})$ and $\mathrm{TiO}$ (Ti). The crystallochemical formulae of garnet were calculated on the basis of 12 oxygen atoms. The crystallochemical formulae of feldspar were recalculated to 8 and those of micas to 22 oxygen atoms. Amphibole formulae were recalculated assuming $23 \mathrm{O}, 2(\mathrm{OH}, \mathrm{F}, \mathrm{Cl})$ and $\mathrm{Fe}^{3+}$ contents were estimated using the recalculation method 13 eCNK (Leake et al. 1997). The abbreviations used are according to Kretz (1983).

Temperatures and pressures were estimated using the Average P-T mode in Thermocalc 3.3 (Holland and Powell 1998, version 3.25, dataset 5.5, 12 November 2004). The correlated uncertainties of $P$ and $T$ allow error ellipses to be plotted (for details see Powell and Holland
1988). The activities and activity uncertainties of endmembers were obtained from AX software (T. Holland and R. Powell, unpublished).

Samples at least 3-4 $\mathrm{kg}$ in weight were used for whole-rock geochemical analyses. Major and trace elements were determined at Acme Analytical Laboratories, Ltd., Vancouver, Canada. Major oxides were analysed by ICP-MS method. Loss on ignition (LOI) was calculated by weight difference after ignition at $1000{ }^{\circ} \mathrm{C}$. The rare earth and trace elements were analyzed by INAA and ICP-MS following a $\mathrm{LiBO}_{2}$ fusion. Geochemical data were recalculated using the GCDkit software package (Janoušek et al. 2006). For comparison were used published analyses of gneisses, granites and the rocks of the tonalite group (Čech ed. 2009; Buriánek ed. 2009).

\section{Petrography}

Augen gneisses are fine- to medium-grained rocks with characteristic lenticular feldspar porphyroclasts or porphyroblasts (Fig. 3a-f). According to the textural features (relicts of igneous textures) and mineralogical characteristics, augen gneisses are divided into augen metagranitoids (deformed and metamorphosed granites, granodiorites, tonalites, diorites and monzodiorites) and migmatitic augen gneisses.

\subsection{Augen metagranitoids}

Biotite to amphibole-biotite augen metagranitoids ranging to orthogneisses, locally with garnet, are typical of the lower subunit of the PU. Augen metagranitoids are associated with partially melted and assimilated metapelites. Elongated enclaves of metapelites several $\mathrm{mm}$ to $\mathrm{cm}$ in size with a mineral assemblage $\mathrm{Bt}+\mathrm{Ms}+\mathrm{Pl}+$ Sil reflect deformation under sub-solidus conditions.

Analogous biotite to amphibole-biotite augen metagranitoids are related to the Miřetín Pluton in the NW part of the PU and its equivalents in a belt between Krouna, Kameničky, Vortová and Stržanov tectonically incorporated in the Svratka Unit and the Moldanubian Zone. Both subtypes have a similar colour index. They are porphyroclastic (Fig. 3b) rocks with medium-grained lepidogranoblastic to granoblastic matrix and usually exhibit parallel alignment of biotite. Plagioclase augen, $2 \mathrm{~mm}$ to $7 \mathrm{~mm}$ in size are wrapped by quartz, biotite, and rarely sillimanite. They consist of quartz (18-36 vol. $\%$ ), plagioclase (26-49\%), K-feldspar (0-19\%), biotite (14-30\%), muscovite (0-7\%), amphibole (0-13\%), garnet $(0-3 \%)$ and rarely sillimanite, the latter present in some samples only (Tab. 1). Apatite, monazite and zircon are common accessories. 

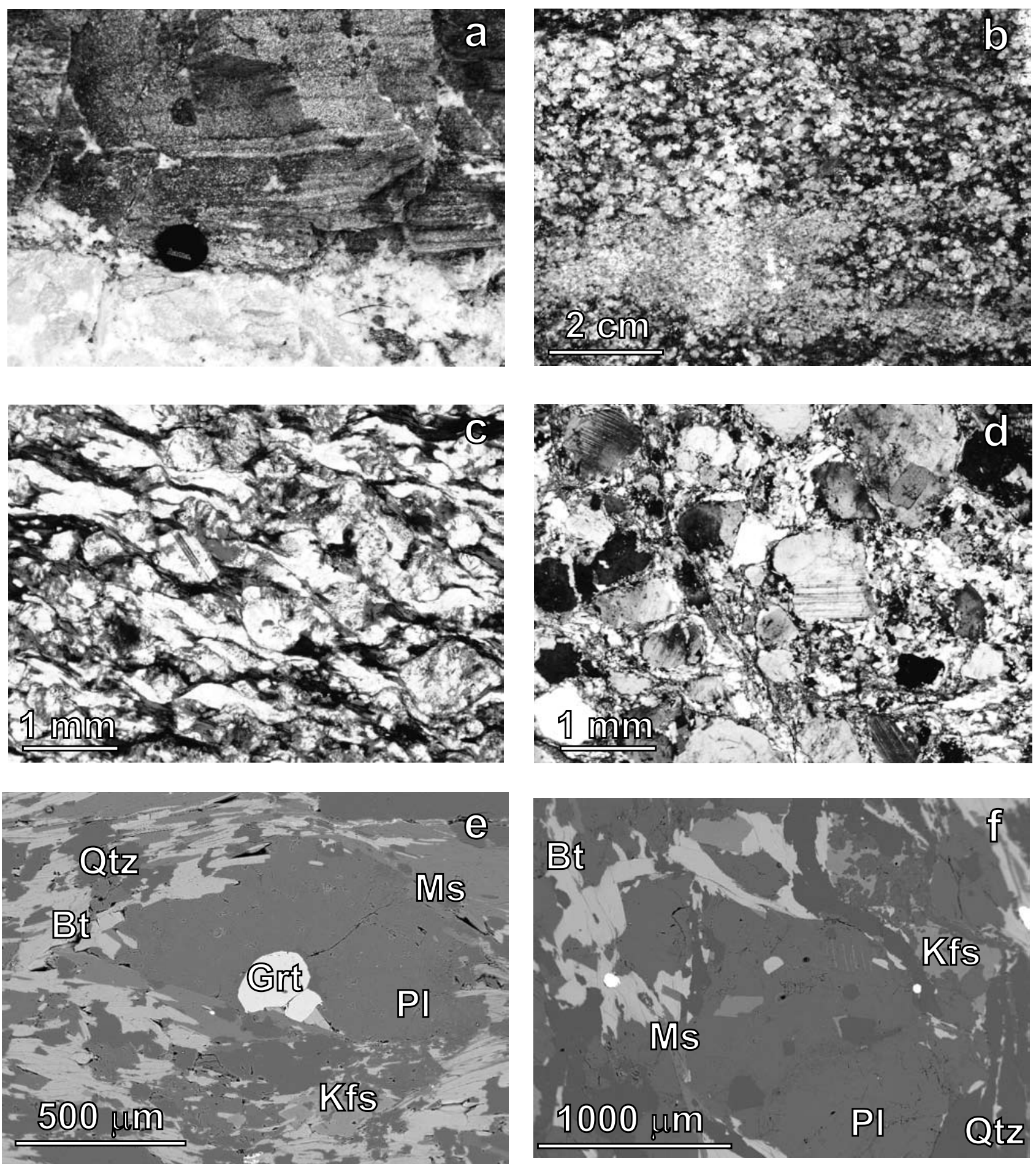

Fig. 3 Typical textures of augen gneisses: a - Contact between marbles and augen gneiss (quarry U vápenky, Bystré); b - Leucocratic augen gneiss, Ubušín (sample 323); c- Migmatitic augen gneiss, Telecí (sample 63, plain polarized light); d - Augen metagranitoid, Krouna (sample 614, crossed nicols); $\mathbf{e}$ - Garnet inclusion in the plagioclase augen from migmatitic augen gneiss, Trhonice (sample 329, BSE image); f - Plagioclase augen from migmatitic augen gneiss, Telecí (sample 63, BSE image).

Quartz occurs as anhedral grains with undulatory extinction. Plagioclase (Tab. 2) porphyroblasts are usually normally zoned with slight oscillations. The cores $\mathrm{An}_{41-47}$ are often strongly sericitized compared to the rims $A_{39-40}$ (sample 197). Plagioclase grains from garnet-biotite metagranitoids (sample 245) exhibit other type of oscillatory zoning $\left(\mathrm{An}_{16-23}\right)$. The $\mathrm{P}_{2} \mathrm{O}_{5}$ content in the plagioclase is variable (up to 0.18 wt. \%, Fig. 4). 
Tab. 1 Localization and modal composition of the studied samples (vol. \%)

\begin{tabular}{|c|c|c|c|c|c|c|c|c|c|c|c|c|c|}
\hline Sample & Locality & Rock & Longitude $\left({ }^{\circ} \mathbf{E}\right)$ & Latitude $\left({ }^{\circ} \mathbf{N}\right)$ & Qtz & PI & Kfs & Bt & Amp & Grt & Sil & Ms & Accessory \\
\hline 63 & Telecí & augen gneiss & $16^{\circ} 10^{\prime} 05.22^{\prime \prime}$ & $49^{\circ} 42^{\prime} 46.42^{\prime \prime}$ & 28 & 41 & 5 & 22 & 0 & 0 & 0 & 4 & $\mathrm{p}+\mathrm{Mnz}$ \\
\hline $1 / 3$ & Borová & augen gneiss & $16^{\circ} 09^{\prime} 09.45^{\prime \prime}$ & $49^{\circ} 43^{\prime} 52.02^{\prime \prime}$ & 30 & 42 & 0 & 25 & 0 & 0 & 0 & 3 & $\mathrm{Ap}+\mathrm{Mnz}+\mathrm{Zrn}+\mathrm{Ttn}$ \\
\hline 68 & Bystré & augen gneiss & $16^{\circ} 20^{\prime} 13.46^{\prime \prime}$ & $49^{\circ} 37^{\prime} 58.57^{\prime \prime}$ & 28 & 24 & 9 & 27 & 0 & 0 & 0 & 12 & $\mathrm{Ap}+\mathrm{Mnz}+\mathrm{Zrn}$ \\
\hline 323 & Ubušín & augen gneiss & $16^{\circ} 16^{\prime} 14.96^{\prime \prime}$ & $49^{\circ} 37^{\prime} 27.25^{\prime \prime}$ & 27 & 38 & 8 & 18 & 0 & 2 & 2 & 5 & $\mathrm{Ap}+\mathrm{Mnz}+\mathrm{Zrn}$ \\
\hline 1614 & Borová & augen gneiss & $16^{\circ} 09^{\prime} 08.44^{\prime \prime}$ & $49^{\circ} 44^{\prime} 09.14^{\prime \prime}$ & 35 & 34 & 7 & 20 & 0 & 3 & 0 & 1 & $\mathrm{Ap}+\mathrm{Mnz}$ \\
\hline 356 & Jedlová & augen gneiss & $16^{\circ} 17^{\prime} 40.99^{\prime \prime}$ & $49^{\circ} 39^{\prime} 44.22^{\prime \prime}$ & 33 & 42 & 5 & 17 & 0 & 0 & 1 & 2 & $\mathrm{Ap}+\mathrm{Mnz}$ \\
\hline 1 & Trhonice-Sedliště & augen gneiss & $16^{\circ} 14^{\prime} 10.00^{\prime \prime}$ & $49^{\circ} 38^{\prime} 44.21^{\prime \prime}$ & 30 & 33 & 17 & 19 & 0 & 0 & 0 & 1 & $\mathrm{Ap}+\mathrm{Mnz}$ \\
\hline 8 & Chlum & augen gneiss & $16^{\circ} 19^{\prime} 08.83^{\prime \prime}$ & $49^{\circ} 34^{\prime} 37.53^{\prime \prime}$ & 35 & 28 & 19 & 18 & 0 & 0 & 0 & & $\mathrm{Ap}+\mathrm{Mnz}$ \\
\hline $323 b$ & Ubušín & augen gneiss & $16^{\circ} 16^{\prime} 14.96^{\prime \prime}$ & $49^{\circ} 37^{\prime} 27.25^{\prime \prime}$ & 33 & 47 & 0 & 17 & 0 & 0 & 0 & 3 & $\mathrm{Ap}+\mathrm{Mnz}$ \\
\hline $328 a$ & Trhonice & augen gneiss & $16^{\circ} 14^{\prime} 57.08^{\prime \prime}$ & $49^{\circ} 37^{\prime} 47.66^{\prime \prime}$ & 33 & 41 & 0 & 16 & 0 & 8 & 0 & 2 & $\mathrm{Ap}+\mathrm{Mnz}$ \\
\hline $328 b$ & Trhonice & augen gneiss & $16^{\circ} 14^{\prime} 57.08^{\prime \prime}$ & $49^{\circ} 37^{\prime} 47.66^{\prime \prime}$ & 30 & 44 & 2 & 20 & 0 & 0 & 0 & 4 & $\mathrm{Ap}+\mathrm{Mnz}$ \\
\hline 329a & Trhonice & augen gneiss & $16^{\circ} 15^{\prime} 02.11^{\prime \prime}$ & $49^{\circ} 37^{\prime} 48.24^{\prime \prime}$ & 32 & 39 & 6 & 18 & 0 & 0 & 0 & 5 & $\mathrm{Ap}+\mathrm{Mnz}$ \\
\hline 329b & Trhonice & augen gneiss & $16^{\circ} 15^{\prime} 02.11^{\prime \prime}$ & $49^{\circ} 37^{\prime} 48.24^{\prime \prime}$ & 28 & 37 & 0 & 20 & 0 & 5 & 0 & 10 & $\mathrm{Ap}+\mathrm{Mnz}$ \\
\hline 245 & Trhonice & metagranitoid & $16^{\circ} 15^{\prime} 30.96^{\prime \prime}$ & $49^{\circ} 38^{\prime} 42.73^{\prime \prime}$ & 25 & 40 & 6 & 29 & 0 & 0 & 0 & 0 & $\mathrm{Ap}+\mathrm{Mnz}$ \\
\hline 128 & Kameničky & metagranitoid & $15^{\circ} 58^{\prime} 23.81^{\prime \prime}$ & $49^{\circ} 44^{\prime} 36.88^{\prime \prime}$ & 33 & 31 & 12 & 21 & 0 & 0 & 0 & 3 & $\mathrm{Ap}+\mathrm{Mnz}+\mathrm{Zrn}+\mathrm{Ttn}$ \\
\hline 129 & Jeníkov & metagranitoid & $15^{\circ} 56^{\prime} 30.37^{\prime \prime}$ & $49^{\circ} 44^{\prime} 30.00^{\prime \prime}$ & 32 & 26 & 17 & 18 & 0 & 0 & 0 & 7 & $\mathrm{Ap}+\mathrm{Mnz}+\mathrm{Zrn}$ \\
\hline 132 & Vortová - U Vily & metagranitoid & $15^{\circ} 56^{\prime} 29.85^{\prime \prime}$ & $49^{\circ} 42^{\prime} 10.41^{\prime \prime}$ & 28 & 42 & 0 & 17 & 13 & 0 & 0 & 0 & $\mathrm{Ap}+\mathrm{Mnz}+\mathrm{Zrn}+\mathrm{Ttn}$ \\
\hline 144 & Otradov & metagranitoid & $16^{\circ} 03^{\prime} 09.29^{\prime \prime}$ & $49^{\circ} 47^{\prime} 07.42^{\prime \prime}$ & 36 & 31 & 19 & 14 & 0 & 0 & 0 & 0 & $\mathrm{Ap}+\mathrm{Mnz}+\mathrm{Zrn}$ \\
\hline $1 / 1$ & Borová & metagranitoid & $16^{\circ} 08^{\prime} 11.22^{\prime \prime}$ & $49^{\circ} 45^{\prime} 16.97 "$ & 30 & 49 & 4 & 15 & 0 & 0 & 0 & 2 & $\mathrm{Ap}+\mathrm{Mnz}+\mathrm{Zrn}$ \\
\hline $1 / 2$ & Borová & metagranitoid & $16^{\circ} 08^{\prime} 19.32^{\prime \prime}$ & $49^{\circ} 44^{\prime} 47.08^{\prime \prime}$ & 25 & 48 & 6 & 18 & 0 & 3 & 0 & 0 & $\mathrm{Ap}+\mathrm{Mnz}+\mathrm{Zrn}$ \\
\hline 225 & Polnička-Stříbrný r. & metagranitoid & $15^{\circ} 54^{\prime} 01.06^{\prime \prime}$ & $49^{\circ} 36^{\prime} 42.00^{\prime \prime}$ & 24 & 36 & 15 & 18 & 7 & 0 & 0 & 0 & $\mathrm{Ap}+\mathrm{Mnz}+\mathrm{Zrn}+\mathrm{Ttn}$ \\
\hline 197 & Stržanov & metagranitoid & $15^{\circ} 55^{\prime} 58.72^{\prime \prime}$ & $49^{\circ} 36^{\prime} 40.20^{\prime \prime}$ & 28 & 42 & 0 & 18 & 12 & 0 & 0 & 0 & $\mathrm{Ap}+\mathrm{Mnz}+\mathrm{Zrn}+\mathrm{Ttn}$ \\
\hline 11 & Korouhev & metagranitoid & $16^{\circ} 17^{\prime} 02.51^{\prime \prime}$ & $49^{\circ} 39^{\prime} 40.37^{\prime \prime}$ & 18 & 47 & 4 & 30 & 0 & 0 & 0 & 1 & $\mathrm{Ap}+\mathrm{Mnz}+\mathrm{Zrn}$ \\
\hline 614 & Krouna & metagranitoid & $16^{\circ} 00^{\prime} 40.14^{\prime \prime}$ & $49^{\circ} 45^{\prime} 45.33^{\prime \prime}$ & 29 & 41 & 5 & 25 & 0 & 0 & 0 & 0 & $\mathrm{Ap}+\mathrm{Mnz}+\mathrm{Zrn}$ \\
\hline 356 & Jedlová & metagranitoid & $16^{\circ} 17^{\prime} 40.99^{\prime \prime}$ & $49^{\circ} 39^{\prime} 44.22^{\prime \prime}$ & 29 & 45 & 9 & 17 & 0 & 0 & 0 & 0 & $\mathrm{Ap}+\mathrm{Mnz}+\mathrm{Zrn}$ \\
\hline 1003 & Bystré & gneiss & $16^{\circ} 21^{\prime} 48.19^{\prime \prime}$ & $49^{\circ} 37^{\prime} 02.12^{\prime \prime}$ & 28 & 27 & 6 & 28 & 0 & 3 & 1 & 7 & $\mathrm{Ap}+\mathrm{Mnz}$ \\
\hline 331 & Nedvězí & gneiss & $16^{\circ} 16^{\prime} 54.34 "$ & $49^{\circ} 37^{\prime} 47.72^{\prime \prime}$ & 27 & 32 & 0 & 25 & 0 & 4 & 2 & 10 & $\mathrm{Ap}+\mathrm{Mnz}$ \\
\hline 14 & Bystré & gneiss & $16^{\circ} 20^{\prime} 29.01^{\prime \prime}$ & $49^{\circ} 36^{\prime} 30.46^{\prime \prime}$ & 30 & 38 & 0 & 26 & 0 & 3 & 1 & 2 & $\mathrm{Ap}+\mathrm{Mnz}$ \\
\hline
\end{tabular}

Tab. 2 Chemical composition of plagioclase (wt. \% and apfu based on 8 O)

\begin{tabular}{|c|c|c|c|c|c|c|c|c|c|c|c|c|}
\hline Sample & $1614 / 2$ & $1614 / 19$ & $1614 / 21$ & $63 / 132$ & $63 / 140$ & $329 / 1$ & $329 / 2$ & $245 / 3$ & $245 / 4$ & $197 / 72$ & 197/89 & $197 / 96$ \\
\hline $\mathrm{SiO}_{2}$ & 62.08 & 64.84 & 63.35 & 61.69 & 64.50 & 62.30 & 63.01 & 62.98 & 64.90 & 58.67 & 58.83 & 56.63 \\
\hline $\mathrm{P}_{2} \mathrm{O}_{5}$ & 0.00 & 0.05 & 0.00 & 0.03 & 0.07 & 0.14 & 0.09 & 0.12 & 0.21 & 0.00 & 0.00 & 0.04 \\
\hline $\mathrm{Al}_{2} \mathrm{O}_{3}$ & 23.59 & 18.77 & 22.98 & 24.05 & 18.45 & 22.42 & 18.35 & 23.44 & 18.81 & 26.32 & 26.26 & 27.65 \\
\hline $\mathrm{FeO}$ & 0.06 & 0.03 & 0.00 & 0.01 & 0.02 & 0.01 & 0.09 & 0.00 & 0.02 & 0.02 & 0.08 & 0.05 \\
\hline $\mathrm{CaO}$ & 4.73 & 0.00 & 4.71 & 5.33 & 0.00 & 4.16 & 0.00 & 4.67 & 0.01 & 8.18 & 7.94 & 9.67 \\
\hline $\mathrm{Na}_{2} \mathrm{O}$ & 9.12 & 1.30 & 8.80 & 8.76 & 1.20 & 9.44 & 1.47 & 8.37 & 1.52 & 6.70 & 6.79 & 5.84 \\
\hline $\mathrm{K}_{2} \mathrm{O}$ & 0.23 & 14.58 & 0.27 & 0.29 & 15.13 & 0.32 & 14.11 & 0.40 & 14.31 & 0.07 & 0.16 & 0.27 \\
\hline $\mathrm{BaO}$ & 0.00 & 0.78 & 0.08 & 0.00 & 0.04 & 0.00 & 1.44 & 0.03 & 0.51 & 0.02 & 0.00 & 0.07 \\
\hline SrO & 0.00 & 0.00 & 0.00 & 0.03 & 0.10 & 0.00 & 0.00 & 0.03 & 0.10 & 0.00 & 0.00 & 0.00 \\
\hline Total & 99.82 & 99.56 & 100.10 & 100.15 & 99.37 & 98.78 & 97.12 & 99.96 & 99.78 & 99.97 & 100.08 & 100.15 \\
\hline $\mathbf{S i}$ & 2.759 & 2.983 & 2.799 & 2.737 & 2.988 & 2.795 & 2.968 & 2.784 & 2.977 & 2.620 & 2.624 & 2.539 \\
\hline Al & 1.236 & 1.018 & 1.197 & 1.258 & 1.007 & 1.185 & 1.019 & 1.221 & 1.017 & 1.385 & 1.381 & 1.461 \\
\hline $\mathrm{Fe}^{3+}$ & 0.002 & 0.001 & 0.000 & 0.000 & 0.001 & 0.000 & 0.004 & 0.000 & 0.001 & 0.001 & 0.003 & 0.002 \\
\hline T-site & 3.997 & 4.002 & 3.996 & 3.995 & 3.996 & 3.981 & 3.991 & 4.005 & 3.995 & 4.007 & 4.008 & 4.002 \\
\hline K & 0.013 & 0.856 & 0.015 & 0.016 & 0.894 & 0.018 & 0.848 & 0.022 & 0.837 & 0.004 & 0.009 & 0.016 \\
\hline $\mathrm{Na}$ & 0.786 & 0.116 & 0.754 & 0.753 & 0.108 & 0.821 & 0.134 & 0.718 & 0.135 & 0.580 & 0.588 & 0.507 \\
\hline $\mathrm{Ca}$ & 0.223 & 0.000 & 0.220 & 0.250 & 0.000 & 0.197 & 0.000 & 0.218 & 0.000 & 0.387 & 0.375 & 0.459 \\
\hline $\mathbf{B a}$ & 0.000 & 0.014 & 0.001 & 0.000 & 0.001 & 0.000 & 0.027 & 0.001 & 0.009 & 0.000 & 0.000 & 0.001 \\
\hline $\mathrm{Sr}$ & 0.000 & 0.000 & 0.000 & 0.001 & 0.003 & 0.000 & 0.000 & 0.001 & 0.003 & 0.000 & 0.000 & 0.000 \\
\hline O-site & 1.022 & 0.986 & 0.990 & 1.021 & 1.006 & 1.037 & 1.009 & 0.959 & 0.984 & 0.971 & 0.971 & 0.983 \\
\hline $\mathrm{K}+\mathrm{Na}+\mathrm{Ca}$ & 1.022 & 0.971 & 0.989 & 1.020 & 1.002 & 1.037 & 0.983 & 0.958 & 0.973 & 0.971 & 0.971 & 0.982 \\
\hline An & 22 & 0 & 22 & 25 & 0 & 19 & 0 & 23 & 0 & 40 & 39 & 47 \\
\hline $\mathbf{A b}$ & 77 & 12 & 76 & 74 & 11 & 79 & 14 & 75 & 14 & 60 & 60 & 52 \\
\hline Or & 1 & 88 & 2 & 2 & 89 & 2 & 86 & 2 & 86 & 0 & 1 & 2 \\
\hline
\end{tabular}




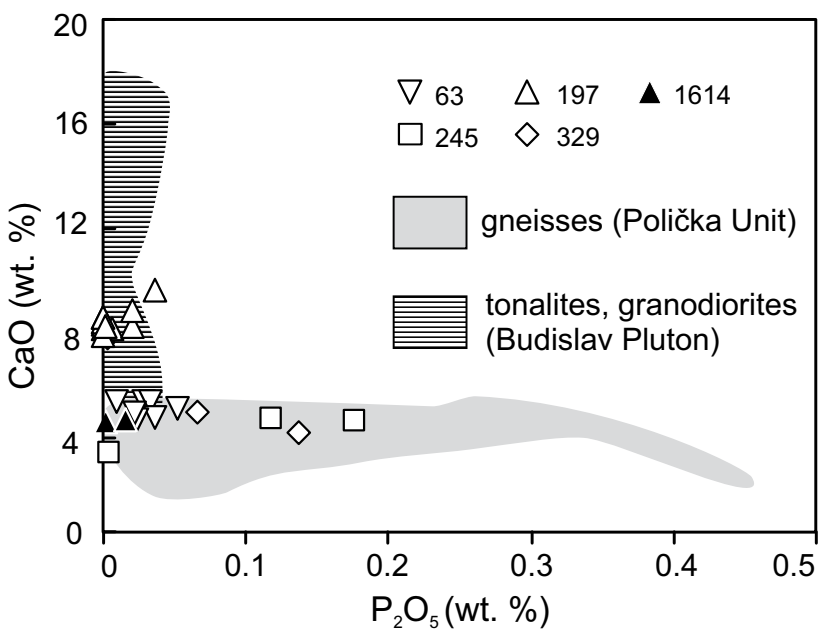

Fig. 4 The $\mathrm{P}_{2} \mathrm{O}_{5}-\mathrm{CaO}$ diagram for the plagioclases from augen gneisses, gneisses and granitoid rocks of the Budislav Pluton. Plagioclases from augen gneisses are subdivided according to the sample number.

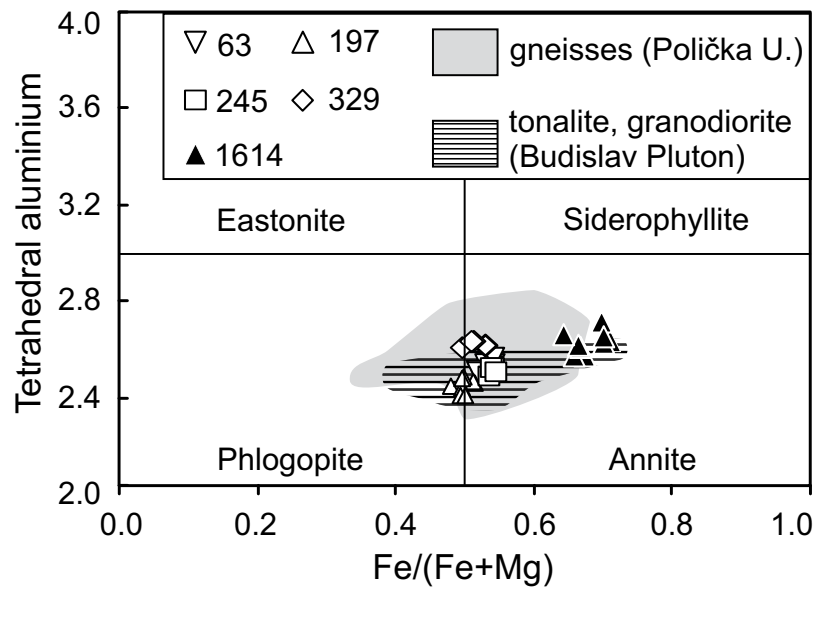

Fig. 5 Classification $\mathrm{Fe} /(\mathrm{Fe}+\mathrm{Mg})$ vs. tetrahedral aluminium diagram of the biotites from augen gneisses, gneisses and the rocks of tonalite group. Biotites from augen gneisses are subdivided according sample number.

Tab. 3 Chemical composition of biotite (wt. \% and apfu based on $22 \mathrm{O}$ )

\begin{tabular}{|c|c|c|c|c|c|c|c|c|c|}
\hline Sample No. & $63 / 41$ & $63 / 45$ & $1614 / 14$ & $1614 / 21$ & $1614 / 26$ & $329 / 30$ & $329 / 31$ & $245 / 8$ & $245 / 10$ \\
\hline $\mathrm{SiO}_{2}$ & 36.27 & 36.21 & 35.03 & 34.49 & 34.71 & 35.44 & 35.60 & 36.70 & 36.62 \\
\hline $\mathrm{TiO}_{2}$ & 2.90 & 3.00 & 0.97 & 2.79 & 2.64 & 3.12 & 1.89 & 3.09 & 3.21 \\
\hline $\mathrm{Al}_{2} \mathrm{O}_{3}$ & 18.32 & 18.94 & 19.12 & 18.40 & 19.40 & 19.11 & 19.36 & 17.75 & 17.86 \\
\hline FeO & 18.55 & 17.91 & 24.28 & 24.57 & 21.84 & 18.08 & 18.95 & 19.42 & 19.59 \\
\hline MnO & 0.25 & 0.29 & 0.63 & 0.66 & 0.47 & 0.23 & 0.24 & 0.28 & 0.35 \\
\hline MgO & 9.63 & 9.26 & 6.62 & 5.62 & 6.78 & 9.59 & 10.16 & 9.35 & 9.11 \\
\hline $\mathrm{Na}_{2} \mathrm{O}$ & 0.10 & 0.09 & 0.05 & 0.06 & 0.08 & 0.16 & 0.15 & 0.12 & 0.12 \\
\hline $\mathrm{K}_{2} \mathrm{O}$ & 9.82 & 9.95 & 9.03 & 9.73 & 10.21 & 9.55 & 9.48 & 9.75 & 9.74 \\
\hline $\mathrm{Cr}_{2} \mathrm{O}_{3}$ & 0.03 & 0.03 & 0.02 & 0.00 & 0.00 & 0.04 & 0.04 & 0.03 & 0.06 \\
\hline $\mathbf{F}$ & - & - & - & - & - & 0.00 & 0.00 & - & - \\
\hline Cl & - & - & - & - & - & 0.01 & 0.02 & - & - \\
\hline $\mathrm{H}_{2} \mathrm{O}^{*}$ & 3.97 & 3.98 & 3.87 & 3.85 & 3.90 & 3.96 & 3.97 & 3.99 & 3.99 \\
\hline $\mathrm{O}=\mathrm{F}, \mathrm{Cl}$ & 0.00 & 0.00 & 0.00 & 0.00 & 0.00 & 0.00 & 0.00 & 0.00 & 0.00 \\
\hline Total & 99.83 & 99.65 & 99.62 & 100.16 & 100.02 & 99.28 & 99.84 & 100.48 & 100.65 \\
\hline$\overline{\mathrm{Si}}$ & 5.474 & 5.460 & 5.431 & 5.364 & 5.338 & 5.368 & 5.374 & 5.521 & 5.506 \\
\hline $\mathbf{A l}^{\mathrm{IV}}$ & 2.526 & 2.540 & 2.569 & 2.636 & 2.662 & 2.632 & 2.626 & 2.479 & 2.494 \\
\hline $\mathbf{A l}^{\mathrm{VI}}$ & 0.733 & 0.825 & 0.925 & 0.737 & 0.855 & 0.778 & 0.819 & 0.669 & 0.671 \\
\hline $\mathbf{T i}$ & 0.329 & 0.341 & 0.113 & 0.326 & 0.306 & 0.355 & 0.214 & 0.350 & 0.363 \\
\hline $\mathrm{Cr}$ & 0.004 & 0.003 & 0.003 & 0.000 & 0.000 & 0.005 & 0.005 & 0.004 & 0.007 \\
\hline $\mathbf{F e}$ & 2.341 & 2.258 & 3.149 & 3.196 & 2.810 & 2.290 & 2.393 & 2.444 & 2.463 \\
\hline Mn & 0.032 & 0.037 & 0.083 & 0.087 & 0.061 & 0.030 & 0.031 & 0.036 & 0.045 \\
\hline Mg & 2.166 & 2.082 & 1.530 & 1.303 & 1.554 & 2.166 & 2.287 & 2.096 & 2.042 \\
\hline $\mathbf{N a}$ & 0.029 & 0.026 & 0.014 & 0.018 & 0.023 & 0.046 & 0.042 & 0.034 & 0.034 \\
\hline $\mathbf{K}$ & 1.891 & 1.914 & 1.786 & 1.930 & 2.002 & 1.844 & 1.826 & 1.871 & 1.868 \\
\hline $\mathbf{F}$ & - & - & - & - & - & 0.003 & 0.004 & - & - \\
\hline Cl & - & - & - & - & - & 0.000 & 0.000 & - & - \\
\hline $\mathrm{OH}^{*}$ & 4.000 & 4.000 & 4.000 & 4.000 & 4.000 & 3.997 & 3.996 & 4.000 & 4.000 \\
\hline Total & 19.525 & 19.486 & 19.608 & 19.597 & 19.610 & 19.515 & 19.621 & 19.506 & 19.495 \\
\hline
\end{tabular}

$* \mathrm{H}_{2} \mathrm{O}$ content calculated on the basis of ideal stoichiometry 
Most of the deformed phenocrysts show typical core and mantle structure defined by large feldspar cores surrounded by newly recrystallized mosaic around the rims. The porphyroblasts also often exhibit variable effects of plastic deformation (e.g., undulatory extinction). Small inclusions of biotite occur predominantly at the rims. $\mathrm{K}$-feldspar $\left(\mathrm{Ab}_{4}\right)$, if present (sample 245), is anhedral and ranges in size from 0.3 to $1.0 \mathrm{~mm}$. Mafic minerals occur as aggregates or in individual grains often concentrated around plagioclase porphyroblasts (Fig. 3c). Amphibole forms fine- to medium-grained, euhedral to subhedral grains. The small columns occasionally show brittle microstructures associated with undulatory extinction. They consist of magnesio-hornblende to pargasite cores $(\mathrm{Si}=6.42-7.49 \mathrm{apfu}, \mathrm{Fe} /(\mathrm{Mg}+\mathrm{Fe})=0.44-0.55)$ and thin actinolite rims $(\mathrm{Si}=7.56 \mathrm{apfu}, \mathrm{Fe} /(\mathrm{Mg}+\mathrm{Fe})=0.68)$. The biotites (Tab. 3) from all of the studied samples fall close to the boundary between annite and phlogopite $\left(\mathrm{Al}^{\mathrm{IV}}=\right.$ $2.40-2.52 \mathrm{apfu}, \mathrm{Fe} /(\mathrm{Mg}+\mathrm{Fe})=0.48-0.55)$ in the classification diagram of Guidotti (1984; Fig. 5).

The mineral chemistry of amphibole-biotite metadiorite from the isolated occurrence of the PU equivalent in the Moldanubian Zone (Stržanov, sample 197) is comparable with chemical composition of the minerals from the rocks of tonalite suite (Figs 4-5). Sillimanite occurs as dense fibrolite or as needle-like inclusions in biotite or quartz. Garnet (Tab. 4) grains are compositionally zoned and characterized by predominant almandine component (Fig. 6) with variable amount of other components $\left(\mathrm{Alm}_{60-72} \mathrm{Grs}_{1-5} \operatorname{Sps}_{11-31} \operatorname{Prp}_{5-17} \mathrm{Adr}_{0-3}\right)$. The grains exhibit

Tab. 4 Representative chemical compositions of garnets and amphiboles (wt. \% and apfu*)

\begin{tabular}{|c|c|c|c|c|c|c|}
\hline Sample & $329 / 27$ & $329 / 39$ & $329 / 40$ & $1614 / 1$ & $1614 / 8$ & $1614 / 4$ \\
\hline $\mathrm{SiO}_{2}$ & 36.99 & 37.02 & 36.71 & 36.98 & 36.11 & 36.53 \\
\hline $\mathrm{TiO}_{2}$ & 0.02 & 0.00 & 0.00 & 0.00 & 0.02 & 0.01 \\
\hline $\mathrm{Al}_{2} \mathrm{O}_{3}$ & 21.19 & 20.90 & 21.03 & 20.97 & 21.10 & 20.80 \\
\hline $\mathrm{Cr}_{2} \mathrm{O}_{3}$ & 0.02 & 0.03 & 0.02 & 0.01 & 0.00 & 0.00 \\
\hline $\mathrm{Fe}_{2} \mathrm{O}_{3}^{\text {calc }}$ & 0.21 & 1.01 & 1.02 & 0.20 & 0.59 & 0.69 \\
\hline $\mathrm{FeO}^{\text {calc }}$ & 29.27 & 30.94 & 30.11 & 31.86 & 29.05 & 29.49 \\
\hline MnO & 8.43 & 4.96 & 5.88 & 6.68 & 10.74 & 10.00 \\
\hline MgO & 2.80 & 4.17 & 4.11 & 2.02 & 1.40 & 1.53 \\
\hline $\mathrm{CaO}$ & 1.48 & 1.55 & 1.49 & 1.70 & 1.82 & 1.84 \\
\hline $\mathrm{Na}_{2} \mathrm{O}$ & 0.03 & 0.01 & 0.04 & 0.03 & 0.02 & 0.01 \\
\hline $\mathbf{P}_{2} \mathbf{O}_{5}$ & 0.03 & 0.02 & 0.04 & 0.04 & 0.00 & 0.00 \\
\hline $\mathrm{Y}_{2} \mathrm{O}_{3}$ & 0.034 & 0.043 & 0.078 & 0 & 0 & 0 \\
\hline Total & 100.51 & 100.66 & 100.52 & 100.48 & 100.83 & 100.89 \\
\hline $\mathbf{S i}$ & 2.974 & 2.957 & 2.939 & 2.988 & 2.934 & 2.961 \\
\hline $\mathbf{P}$ & 0.002 & 0.002 & 0.002 & 0.003 & 0.000 & 0.000 \\
\hline $\mathbf{T i}$ & 0.001 & 0.000 & 0.000 & 0.000 & 0.001 & 0.000 \\
\hline$\Sigma T$ & 2.978 & 2.959 & 2.942 & 2.991 & 2.935 & 2.962 \\
\hline Al & 2.008 & 1.968 & 1.985 & 1.996 & 2.021 & 1.987 \\
\hline $\mathbf{T i}$ & 0.000 & 0.000 & 0.000 & 0.000 & 0.000 & 0.000 \\
\hline $\mathrm{Fe}^{3+}$ & 0.013 & 0.061 & 0.062 & 0.012 & 0.036 & 0.042 \\
\hline $\mathrm{Cr}$ & 0.001 & 0.002 & 0.001 & 0.001 & 0.000 & 0.000 \\
\hline Mg & 0.335 & 0.497 & 0.491 & 0.244 & 0.170 & 0.184 \\
\hline $\mathrm{Fe}^{2+}$ & 1.968 & 2.067 & 2.016 & 2.152 & 1.975 & 2.000 \\
\hline Mn & 0.574 & 0.336 & 0.399 & 0.457 & 0.739 & 0.687 \\
\hline $\mathrm{Ca}$ & 0.128 & 0.133 & 0.128 & 0.147 & 0.158 & 0.160 \\
\hline $\mathbf{N a}$ & 0.008 & 0.002 & 0.009 & 0.007 & 0.004 & 0.003 \\
\hline $\mathbf{Y}$ & 0.001 & 0.002 & 0.003 & 0.000 & 0.000 & 0.000 \\
\hline$\Sigma$ Cat. & 8.013 & 8.026 & 8.036 & 8.007 & 8.038 & 8.025 \\
\hline Alm & 65 & 67 & 65 & 72 & 64 & 65 \\
\hline Adr & 1 & 3 & 3 & 1 & 2 & 2 \\
\hline Grs & 4 & 1 & 1 & 4 & 4 & 3 \\
\hline Prp & 11 & 17 & 17 & 8 & 6 & 6 \\
\hline Sps & 19 & 11 & 14 & 15 & 25 & 23 \\
\hline
\end{tabular}

\begin{tabular}{|c|c|c|c|}
\hline Amphibole & & & \\
\hline Sample & $197 / 63$ & $197 / 75$ & $197 / 88$ \\
\hline $\mathrm{SiO}_{2}$ & 50.97 & 42.49 & 51.46 \\
\hline $\mathrm{TiO}_{2}$ & 0.30 & 1.20 & 0.26 \\
\hline $\mathrm{Al}_{2} \mathrm{O}_{3}$ & 4.95 & 12.79 & 4.30 \\
\hline $\mathrm{Cr}_{2} \mathrm{O}_{3}$ & 0.02 & 0.05 & 0.01 \\
\hline $\mathrm{Fe}_{2} \mathrm{O}_{3}^{\text {calc }}$ & 1.57 & 1.01 & 1.14 \\
\hline $\mathrm{FeO}^{\text {calc }}$ & 13.07 & 15.84 & 12.78 \\
\hline MnO & 0.57 & 0.55 & 0.46 \\
\hline MgO & 13.40 & 8.95 & 13.96 \\
\hline $\mathrm{CaO}$ & 12.02 & 11.56 & 12.50 \\
\hline $\mathrm{Na}_{2} \mathrm{O}$ & 0.59 & 1.40 & 0.40 \\
\hline $\mathbf{K}_{2} \mathbf{O}$ & 0.28 & 1.47 & 0.23 \\
\hline $\mathrm{H}_{2} \mathrm{O}$ & 2.07 & 2.00 & 2.08 \\
\hline Total & 99.82 & 99.31 & 99.58 \\
\hline $\mathbf{S i}$ & 7.421 & 6.416 & 7.489 \\
\hline $\mathbf{A l}^{\mathrm{IV}}$ & 0.579 & 1.584 & 0.511 \\
\hline$\Sigma T$ & 8.000 & 8.000 & 8.000 \\
\hline $\mathbf{A l}^{\mathrm{VI}}$ & 0.271 & 0.692 & 0.228 \\
\hline $\mathbf{T i}$ & 0.032 & 0.136 & 0.028 \\
\hline $\mathbf{F e}^{3+}$ & 0.172 & 0.115 & 0.124 \\
\hline $\mathrm{Cr}$ & 0.003 & 0.006 & 0.002 \\
\hline Mg & 2.910 & 2.014 & 3.029 \\
\hline $\mathrm{Fe}^{2+}$ & 1.592 & 1.999 & 1.555 \\
\hline Mn & 0.021 & 0.037 & 0.033 \\
\hline$\Sigma C$ & 5.000 & 5.000 & 5.000 \\
\hline Mn & 0.049 & 0.033 & 0.024 \\
\hline $\mathrm{Ca}$ & 1.876 & 1.870 & 1.949 \\
\hline $\mathbf{N a}$ & 0.076 & 0.097 & 0.027 \\
\hline$\Sigma \mathbf{B}$ & 2.000 & 2.000 & 2.000 \\
\hline $\mathrm{Na}$ & 0.092 & 0.313 & 0.086 \\
\hline $\mathbf{K}$ & 0.052 & 0.284 & 0.042 \\
\hline $\mathbf{\Sigma A}$ & 0.144 & 0.596 & 0.127 \\
\hline $\mathbf{M g} / \mathbf{F e}+\mathbf{M g}$ & 0.646 & 0.502 & 0.661 \\
\hline$\Sigma$ Cat. & 15.144 & 15.596 & 15.127 \\
\hline
\end{tabular}

* garnet formulae calculated on the basis of $12 \mathrm{O}$, formulae of amphiboles based on $23 \mathrm{O}$ ( $\mathrm{Cl}, \mathrm{F}$ below detection limit) 


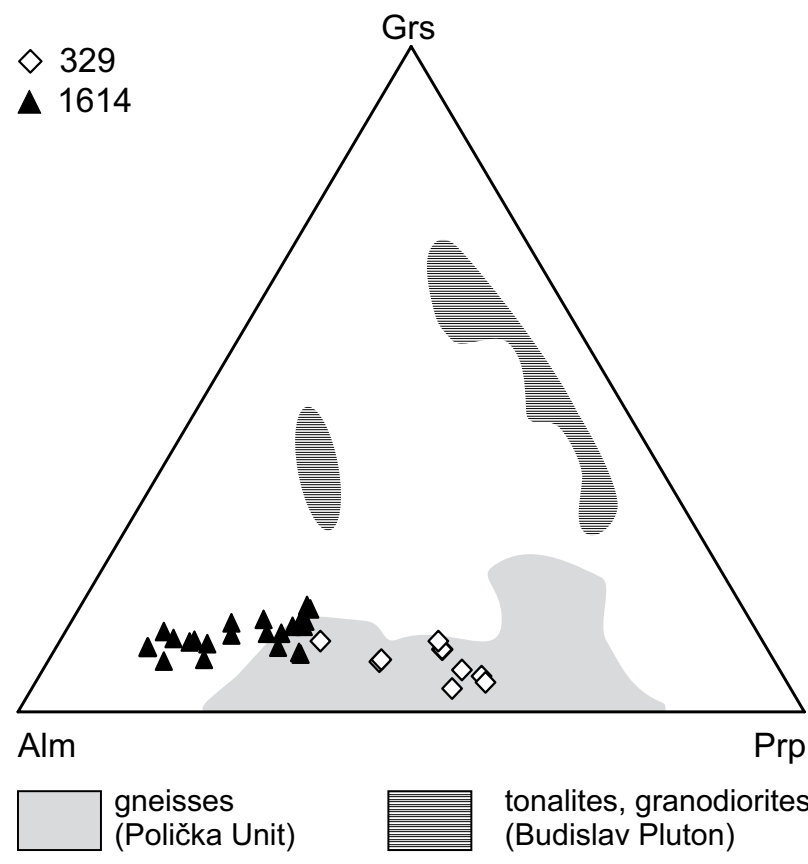

Fig. 6 Ternary Sps-Grs-Prp plot for garnets from augen gneisses, gneisses and rocks of the Budislav Pluton.

a decrease in $\mathrm{Fe}, \mathrm{Mg}$ and increase in $\mathrm{Mn},[\mathrm{Fe} /(\mathrm{Fe}+\mathrm{Mg})]$ from core to rim. The almandine-poor and spessartinerich rims are believed to have developed during retrogression. Garnet inclusions inside the plagioclase augen show reverse zoning with slightly increasing Alm and decreasing Sps components from core to rim. Similar compositions of plagioclase, garnet and biotite (Figs 4-5) were observed in biotite augen gneisses and in the surrounding gneisses and migmatites.

\subsection{Migmatitic augen gneisses}

Migmatitic augen gneisses usually border the bodies and layers of augen metagranitoids or form separate layers several $\mathrm{cm}$ to several $\mathrm{m}$ thick (Fig. 3a). Migmatitic augen gneisses are medium- to coarse-grained, lepidograno- blastic, consisting of plagioclase (24-47 vol. \%), quartz (27-35\%), biotite (16-27\%), K-feldspar (0-19\%). Muscovite (1-12\%), garnet (0-8\%) or sillimanite (0-2\%) are present in some cases. Accessoric apatite, monazite, xenotime and zircon are common. Plagioclases occur as small euhedral crystals in the groundmass, as inclusions in K-feldspars or larger porphyroblasts up to $1 \mathrm{~cm}$ in diameter (Fig. 3f). Small plagioclases are relatively homogeneous in chemical composition $\left(\mathrm{An}_{22}\right.$ to $\left.\mathrm{An}_{20}\right)$. Large oval-shaped porphyroclasts of plagioclase are characterized by a complex zoning. Relatively sodic $\left(\mathrm{An}_{16-21}\right)$ cores are surrounded by slightly more calcic rims $\left(\mathrm{An}_{22-25}\right)$. The oval-shaped plagioclases commonly show evidence of a weak ductile deformation (undulatory extinction). Less common anhedral $\mathrm{K}$-feldspars $\left(\mathrm{Ab}_{11-12}\right)$ are usually perthitic, with myrmekite intergrowths at the contacts with plagioclase. The larger plagioclase porphyroblasts usually contain inclusions of biotite, quartz, muscovite and apatite. Quartz forms anhedral subgrains, recrystallized grains and/or ribbons (Fig. 3c-d). Locally chloritized biotite forms subhedral flakes. According to the classification diagram by Guidotti (1984), the biotites belong to annite $\left(\mathrm{Al}^{\mathrm{IV}}=2.50-2.48\right.$ apfu, $\left.\mathrm{Fe} /(\mathrm{Mg}+\mathrm{Fe})=0.52-0.71\right)$. Muscovite, if present, is generally lath-shaped. In some cases it replaces K-feldspar; more commonly it occurs as inclusions within other minerals (e. g. plagioclase). Micas-rich bands anastomose around plagioclase porphyroclasts and in strongly deformed samples often define S-C fabrics.

Biotite and muscovite-biotite gneisses are locally migmatitized in the vicinity of migmatitic augen gneisses. The gneisses enclose $1-2 \mathrm{~cm}$ thick parallel felsic layers formed by a clearly defined leucosome. The leucosome contains plagioclase, quartz and small amount muscovite and biotite; K-feldspar is subordinate or fully absent. The mafic parts are biotite-rich, consisting of $\mathrm{Qtz}+\mathrm{Bt}+\mathrm{Pl} \pm$ $\mathrm{Ms} \pm \mathrm{Sil} \pm$ Grt assemblage. Both mafic and felsic layers are medium-grained, with granoblastic to lepidogranoblastic textures. Contacts with augen gneisses are usually fuzzy.

\subsection{Estimation of P-T conditions of metamorphism}

Tab. 5 Results of P-T calculations

\begin{tabular}{lllccccc}
\hline Sample & Rock & avT & sd(T) & avP & sd(P) & corr & sigfit \\
\hline $\mathbf{1 6 1 4}$ & augen gneiss & 795 & 33 & 7.0 & 1.0 & 0.773 & 0.96 \\
$\mathbf{3 2 9}$ & augen gneiss & 755 & 22 & 6.9 & 0.9 & 0.713 & 0.96 \\
$\mathbf{3 2 9 b}$ & augen gneiss & 754 & 22 & 7.1 & 0.9 & 0.730 & 0.91 \\
$\mathbf{1 4}$ & Ms-Bt gneiss & 675 & 35 & 7.0 & 1.4 & 0.729 & 0.99 \\
$\mathbf{3 3 1}$ & Ms-Bt gneiss & 654 & 29 & 6.7 & 1.2 & 0.910 & 0.64 \\
$\mathbf{1 0 0 3}$ & Ms-Bt gneiss & 714 & 85 & 8.8 & 1.4 & 0.803 & 0.62 \\
$\mathbf{8 7 *}$ & Ms-Bt gneiss & 640 & 30 & 5.7 & 1.2 & 0.919 & 0.60 \\
\hline
\end{tabular}

The average $\mathrm{T}\left({ }^{\circ} \mathrm{C}\right)(\mathrm{avT})$ and $\mathrm{P}(\mathrm{avP})(\mathrm{kbar})$, the standard deviation (sd), the error correlation coefficient (corr) and sigfit are calculated by THERMOCALC.

* gneiss from middle subunit PU (Korouhev)
The low-variance matrix assemblage is supposed to preserve peak metamorphic conditions (after melt crystallization) in migmatitic augen gneisses (metamorphic event $\mathrm{M}_{2}$ ): $\mathrm{Pl}+$ $\mathrm{Bt}+\mathrm{Kfs}+\mathrm{Qtz} \pm \mathrm{Grt} \pm \mathrm{Sil} \pm$ Ilm (Tab. 5). For a pure $\mathrm{H}_{2} \mathrm{O}$ fluid equilibrating with the solid assemblage of the garnetbiotite augen gneisses, THERMOCALC gives P-T estimations 


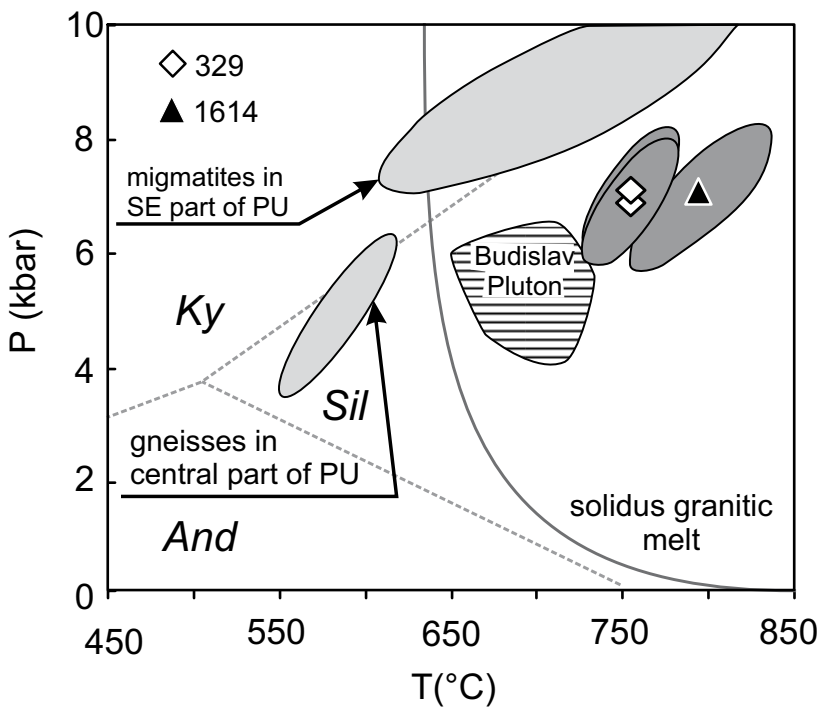

gneisses, migmatites granodiorites

augen gneisses

Fig. 7 The P-T plot for selected igneous and metamorphic rocks of the Polička Unit, metamorphic conditions were calculated using THERMOCALC 3. 3. The position of the $\mathrm{Al}_{2} \mathrm{SiO}_{5}$ triple point from Holdaway and Mukhopadhyay (1993). Conditions of metamorphism or magmatic crystallization for the rocks of Budislav Pluton are from Buriánek et al. (2003) and Buriánek ed. (2009).

of $795 \pm 33{ }^{\circ} \mathrm{C}$ and $7.0 \pm 1.0 \mathrm{kbar}$ with $755 \pm 22{ }^{\circ} \mathrm{C}$ and $6.9 \pm 0.9 \mathrm{kbar}$ (samples 1614 and 329). This calculation is based largely on equilibria involving garnet, biotite, K-feldspar, plagioclase, and quartz (Fig. 7). Sillimanite was totally or partly replaced by muscovite according to metamorphic reaction $\mathrm{Kfs}+\mathrm{Sil}+\mathrm{H}_{2} \mathrm{O}=\mathrm{Ms}+$ Qtz during retrogression $\left(\mathrm{M}_{3}\right)$.

Average P-T conditions of metamorphism $\left(\mathrm{M}_{2}\right)$ calculated for locally migmatitized paragneisses (stromatites) with association $\mathrm{Pl}+\mathrm{Bt}+\mathrm{Ms}+\mathrm{Qtz}+\mathrm{Sil}+\mathrm{Grt}$ from the lower subunit PU are $650-680{ }^{\circ} \mathrm{C}$ and 7 kbar. Geothermobarometry for migmatitized paragneiss sample with mineral assemblage $\mathrm{Pl}+\mathrm{Bt}+\mathrm{Ms}+\mathrm{Qtz}+\mathrm{Kfs}+\mathrm{Sil}+$ Grt from SE part of PU yields $714 \pm 85^{\circ} \mathrm{C}$ and $8.8 \pm$ 1.4 kbar.

Paragneiss from the middle subunit of PU (sample 87) provides significantly lower P-T conditions of c. $600{ }^{\circ} \mathrm{C}$ and $6 \mathrm{kbar}$ for the metamorphic event $\mathrm{M}_{2}$.

\section{Deformation}

The regional metamorphic foliation in metasedimentary rocks shows comparable orientation in the major part of the PU between the Vír dam in the south and Proseč in the north (Fig. 2). Dominating foliation with NW-SE strike and steep to moderate dip to NE is the same in the augen metagranitoids as well as in augen migmatitic gneisses; it bears NW-SE plunging lineation. The mineral lineation in the migmatitic augen gneisses is defined by elongated quartz and feldspars grains together with the orientation of the long axes of biotite plates and sillimanite needles. Kinematic indicators suggest overthrusting in the NW direction.

Typical of the NW part of the unit built by deformed granodiorites and tonalites of the Miřetín Pluton is foliation with steep to moderate dips to the WNW and dipslip stretching lineations. The rocks in the NNE-SSW elongated Miřetín Pluton preserve two distinct solid-state fabrics (Vondrovic and Verner 2008). First, pervasive high-temperature solid-state fabric $\left(D_{1}\right)$ is defined by the ductile deformed biotite and partly recrystallized quartzfeldspathic aggregates. Second, distinct low-temperature solid-state fabric $\left(\mathrm{D}_{2}\right)$ is developed as a spaced cleavage. This fabric is probably connected with normal faulting along the tectonic contact with the Hlinsko Unit.

Magmatic (relics of plagioclase phenocrysts up to $3 \mathrm{~cm}$ in size) and high-temperature solid-state fabric $\left(D_{1}\right)$ in the granodioritic augen gneisses from the NW part of PU (Stržanov, Vortová etc.) are only rarely preserved due to pervasive overprinting by solid-state deformation at moderate to low temperatures.

Several metres thick mylonitic zones concordant with metamorphic fabric are often located near the contact with the Svratka Unit. In the mylonitic zones, original mica and quartz ribbons wrap around euhedral plagioclase phenocrysts. The most common deformational feature observed in the plagioclase is a core-and-mantle structure (White 1975). Quartz grains usually show coarsely sutured grain boundaries.

Deformation of migmatitic augen gneisses under moderate temperatures $\left(c .650{ }^{\circ} \mathrm{C}\right)$ is indicated by a widespread grain boundary migration and recrystallization. Rarely present chessboard pattern in quartz grains, myrmekites on the margins of the K-feldspar grains and quartz ribbons resulted during this deformation stage. The S-C fabrics, accented by the arrangement of micas, developed during the subsequent stages.

The mylonitic foliation is in some cases defined by compositional layering with alternating fine-grained biotite-rich layers and quartz-feldspar lenses or plagioclase porphyroblasts. The lack of internal deformation in the rounded feldspar augen suggests that the strain was likely accommodated in the mica-rich matrix. Plagioclases are often separated in augen with recrystallized tails. Deformation of quartz grains commonly resulted in the quartz core-and-mantle aggregates and quartz ribbons development (Boullier and Bouchez 1978). Large quartz grains exhibit undulatory extinction. Most flakes of biotite or of muscovite show preferred orientation parallel to the main foliation of the rock. 
Tab. 6 Representative whole-rock chemical analyses

\begin{tabular}{|c|c|c|c|c|c|c|c|c|c|c|c|c|}
\hline Sample No. & 63 & $1 / 3$ & 328 & 329 & 128 & 129 & 132 & 144 & $1 / 1$ & $1 / 2$ & 225 & 245 \\
\hline \multicolumn{13}{|l|}{ wt. \% } \\
\hline $\mathrm{SiO}_{2}$ & 69.71 & 70.38 & 73.8 & 66.58 & 64.98 & 70.34 & 59.3 & 63.79 & 64.51 & 64.04 & 58.03 & 66.08 \\
\hline $\mathrm{TiO}_{2}$ & 0.44 & 0.37 & 0.29 & 0.67 & 0.81 & 0.44 & 0.81 & 0.63 & 0.77 & 0.74 & 0.81 & 0.72 \\
\hline $\mathrm{Al}_{2} \mathrm{O}_{3}$ & 14.76 & 14.71 & 13.72 & 15.36 & 15.16 & 14.76 & 16.56 & 15.78 & 16.54 & 16.18 & 17.57 & 15.15 \\
\hline $\mathrm{Fe}_{2} \mathrm{O}_{3}$ & 3.56 & 3.37 & 1.73 & 5.32 & 5.96 & 3.18 & 6.97 & 5.46 & 5.94 & 6.12 & 6.74 & 5.32 \\
\hline MnO & 0.06 & 0.06 & 0.03 & 0.08 & 0.07 & 0.06 & 0.12 & 0.10 & 0.07 & 0.10 & 0.11 & 0.06 \\
\hline $\mathrm{Cr}_{2} \mathrm{O}_{3}$ & b. d. 1. & 0.005 & 0.001 & 0.012 & b. d. 1 . & b. d. 1 . & b. d. 1 . & b. d. 1 . & 0.013 & 0.015 & 0.011 & 0.011 \\
\hline MgO & 1.24 & 0.87 & 0.4 & 2.39 & 2.63 & 1.08 & 3.25 & 2.22 & 2.44 & 2.5 & 3.53 & 2.26 \\
\hline $\mathrm{CaO}$ & 1.17 & 1.24 & 1.23 & 1.59 & 1.99 & 1.95 & 5.38 & 3.40 & 1.63 & 0.99 & 5.21 & 1.26 \\
\hline $\mathrm{Na}_{2} \mathrm{O}$ & 3.55 & 3.58 & 3.75 & 2.64 & 3.44 & 3.70 & 3.43 & 3.42 & 3.13 & 2.34 & 3.41 & 3.08 \\
\hline $\mathrm{K}_{2} \mathrm{O}$ & 4.07 & 4.35 & 4.28 & 3.57 & 3.20 & 2.85 & 2.36 & 4.02 & 3.50 & 2.93 & 2.54 & 4.79 \\
\hline $\mathbf{P}_{2} \mathrm{O}_{5}$ & 0.11 & 0.11 & 0.07 & 0.17 & 0.18 & 0.13 & 0.21 & 0.17 & 0.14 & 0.14 & 0.24 & 0.13 \\
\hline $\mathrm{H}_{2} \mathrm{O}+$ & 0.79 & n. d. & n. d. & n. d. & 0.98 & 0.77 & 0.84 & b. d. 1 . & n. d. & n. d. & n. d. & n. d. \\
\hline $\mathrm{H}_{2} \mathrm{O}-$ & 0.18 & n. d. & n. d. & n. d. & 0.13 & 0.17 & 0.18 & 0.17 & n. d. & n. d. & n. d. & n. d. \\
\hline LOI & n. d. & 1 & 0.6 & 1.5 & n. d. & n. d. & n. d. & n. d. & 1.3 & 3.8 & 1.5 & 0.7 \\
\hline Total & 99.64 & 100.05 & 99.90 & 99.89 & 99.53 & 99.44 & 99.41 & 99.86 & 99.99 & 99.90 & 99.71 & 99.57 \\
\hline \multicolumn{13}{|l|}{ ppm } \\
\hline $\mathbf{B a}$ & 887.4 & 849.7 & 841.1 & 763.2 & 660.6 & 600.4 & 1066.8 & 1110.0 & 813.2 & 485.5 & 972.6 & 854.5 \\
\hline Co & 4.2 & 4.2 & 1.9 & 12.6 & 17.7 & 5.4 & 17.0 & 11.7 & 12.0 & 10.7 & 18.0 & 11.1 \\
\hline $\mathrm{Cu}$ & 8.0 & 23.2 & 5.5 & 37.2 & 37.4 & 10.1 & 10.0 & 17.6 & 19.0 & 25.7 & 25.5 & 22.8 \\
\hline $\mathbf{N i}$ & 9.0 & 12.8 & 3.1 & 25.7 & 37.7 & 9.1 & 16.3 & 21.4 & 28.3 & 47.8 & 21.7 & 28.4 \\
\hline $\mathbf{R b}$ & 149.3 & 141.3 & 151.3 & 123.1 & 170.4 & 123.2 & 124.6 & 124.8 & 176.9 & 113.3 & 122.7 & 206.9 \\
\hline $\mathrm{Sr}$ & 105.9 & 112.3 & 122.8 & 135.2 & 170.2 & 141.0 & 365.8 & 198.7 & 147.2 & 77.5 & 440.8 & 164.0 \\
\hline $\mathbf{V}$ & 27 & 21 & 14 & 99 & 106 & 37 & 137 & 81 & 102 & 113 & 146 & 84 \\
\hline Sn & 4 & 5 & 7 & 7 & 4 & 3 & 3 & 2 & 6 & 3 & 3 & 3 \\
\hline Zn & 79 & 61 & 41 & 109 & 101 & 45 & 68 & 90 & 88 & 65 & 68 & 106 \\
\hline As & 2.1 & 1.9 & 0.6 & 3.0 & 2.4 & 3.5 & 2.9 & 5.4 & 3.9 & 52.8 & 2.4 & 0.6 \\
\hline $\mathbf{U}$ & 3.8 & 4.6 & 5.5 & 3.3 & 3.2 & 4.3 & 2.6 & 2.3 & 3.9 & 2.9 & 12.1 & 3.9 \\
\hline Nb & 9.7 & 10.3 & 7.0 & 11.8 & 13.7 & 7.6 & 11.7 & 13.6 & 12.3 & 10.6 & 11.7 & 12.1 \\
\hline Mo & 0.8 & 0.6 & 0.2 & 1.6 & 1.5 & 0.5 & 1.1 & 1.8 & 0.5 & 2.8 & 1.2 & 0.8 \\
\hline $\mathbf{Y}$ & 46.8 & 53.5 & 36.6 & 31.4 & 23.7 & 24.6 & 18.7 & 23.2 & 31.7 & 26.8 & 24.2 & 28.7 \\
\hline $\mathbf{Z r}$ & 199.3 & 199.6 & 149.7 & 173.8 & 223.8 & 199.2 & 226.6 & 262.0 & 226.3 & 172.5 & 228.4 & 226.7 \\
\hline $\mathbf{P b}$ & 9.4 & 3.5 & 7.2 & 18.0 & 1.5 & 2.9 & 2.1 & 5.8 & 2.7 & 10.1 & 5.4 & 2.9 \\
\hline Cd & $<0.1$ & 0.5 & 0.1 & 0.4 & 0.1 & $<0.1$ & 0.1 & 0.1 & 1.4 & 1.3 & 0.1 & 0.1 \\
\hline Cs & 3.4 & 4.6 & 5.0 & 5.1 & 5.3 & 4.0 & 4.1 & 4.5 & 10.1 & 4.5 & 6.8 & 3.7 \\
\hline Th & 19.1 & 17.4 & 19.0 & 12.4 & 12.5 & 9.5 & 10.9 & 16.4 & 10.7 & 8.9 & 8.6 & 18.7 \\
\hline Ta & 0.8 & 0.7 & 1.0 & 1.0 & 0.9 & 0.8 & 0.7 & 0.8 & 1.0 & 1.0 & 0.8 & 0.8 \\
\hline Hf & 6.8 & 6.2 & 5.5 & 4.4 & 7.5 & 6.3 & 6.6 & 7.6 & 6.4 & 4.6 & 5.9 & 6.9 \\
\hline Sc & n. d. & 9 & 5 & 14 & n. d. & n. d. & n. d. & n. d. & 15 & 17 & 17 & 14 \\
\hline Sb & $<0.1$ & 0.1 & 0.1 & 0.2 & 0.1 & 0.1 & 0.1 & 0.4 & 0.1 & 0.1 & 0.2 & 0.1 \\
\hline Ag & $<0.1$ & 0.3 & $<0.1$ & 0.1 & 0.1 & $<0.1$ & $<0.1$ & 0.1 & 0.1 & 0.1 & $<0.1$ & $<0.1$ \\
\hline Hg & 0.01 & 0.01 & $<0.01$ & 0.02 & 0.02 & $<0.01$ & $<0.01$ & 0.01 & 0.01 & 0.01 & $<0.01$ & $<0.01$ \\
\hline Tl & 0.5 & 0.4 & 0.5 & 0.6 & 0.8 & 0.3 & 0.6 & 0.5 & 0.8 & 0.2 & 0.7 & 0.8 \\
\hline $\mathbf{B i}$ & 0.2 & 0.2 & 0.1 & 0.2 & 0.1 & 0.1 & $<0.1$ & 0.1 & 0.2 & 0.2 & 0.1 & 0.1 \\
\hline $\mathbf{W}$ & 1.4 & 2.7 & 0.7 & 5.6 & 1.5 & 1.5 & 0.4 & 1.0 & 3.7 & 2.1 & 0.5 & 0.9 \\
\hline $\mathbf{G a}$ & 20.0 & 19.4 & 16.5 & 19.6 & 22.3 & 18.7 & 21.9 & 21.7 & 22.0 & 21.3 & 21.2 & 19.6 \\
\hline Se & n. d. & 0.5 & $<0.5$ & $<0.5$ & n. d. & n. d. & n. d. & n. d. & 0.5 & 0.5 & $<0.5$ & $<0.5$ \\
\hline La & 40.1 & 43.3 & 20.3 & 40.2 & 34.9 & 28.8 & 39.6 & 45.8 & 34.5 & 30.4 & 31.9 & 48.1 \\
\hline $\mathrm{Ce}$ & 89.0 & 96.8 & 59.7 & 83.2 & 74.1 & 63.0 & 77.4 & 84.0 & 72.1 & 57.2 & 66.1 & 96.4 \\
\hline Pr & 9.84 & 10.88 & 5.09 & 9.33 & 8.41 & 7.05 & 7.80 & 8.41 & 7.88 & 6.45 & 8.27 & 10.59 \\
\hline Nd & 40.20 & 39.80 & 17.80 & 34.30 & 33.40 & 28.70 & 30.00 & 35.80 & 29.70 & 23.90 & 33.10 & 39.50 \\
\hline Sm & 8.30 & 9.10 & 4.40 & 6.40 & 6.20 & 5.20 & 4.50 & 5.90 & 5.70 & 5.20 & 6.25 & 7.40 \\
\hline $\mathbf{E u}$ & 0.89 & 0.79 & 0.69 & 1.12 & 1.06 & 0.97 & 1.25 & 1.12 & 1.09 & 1.01 & 1.24 & 1.26 \\
\hline Gd & 8.45 & 8.10 & 4.14 & 5.52 & 5.74 & 4.84 & 3.78 & 4.58 & 5.38 & 4.37 & 4.91 & 6.06 \\
\hline Tb & 1.31 & 1.45 & 0.98 & 0.98 & 0.87 & 0.76 & 0.57 & 0.74 & 0.89 & 0.72 & 0.82 & 0.90 \\
\hline Dy & 8.26 & 8.59 & 6.07 & 5.63 & 4.83 & 4.49 & 3.30 & 4.00 & 5.09 & 4.38 & 4.59 & 5.15 \\
\hline Но & 1.56 & 1.75 & 1.20 & 0.97 & 0.86 & 0.81 & 0.54 & 0.74 & 1.06 & 0.90 & 0.83 & 1.01 \\
\hline Er & 4.35 & 5.43 & 3.38 & 2.86 & 2.18 & 2.47 & 1.63 & 2.14 & 3.16 & 2.70 & 2.18 & 2.54 \\
\hline Tm & 0.69 & 0.78 & 0.59 & 0.41 & 0.28 & 0.39 & 0.26 & 0.28 & 0.48 & 0.47 & 0.34 & 0.37 \\
\hline Yb & 4.40 & 4.76 & 3.96 & 2.75 & 1.84 & 2.89 & 2.03 & 2.10 & 3.06 & 2.71 & 1.92 & 2.40 \\
\hline$\underline{\mathbf{L u}}$ & 0.59 & 0.68 & 0.53 & 0.37 & 0.23 & 0.40 & 0.29 & 0.31 & 0.48 & 0.40 & 0.29 & 0.41 \\
\hline
\end{tabular}

b. d. 1. - below detection limit, n. d. - not determined 


\section{Geochemistry}

The whole-rock geochemical data are summarised in Tab. 6. Augen metagranitoids and migmatitic augen gneisses are nearly of the same chemical composition and show $\mathrm{SiO}_{2}$ ranging from 58 to 70 wt. \%. Amphibole-bearing samples have lower $\mathrm{SiO}_{2}$ contents (58 to 64 wt. \%) than biotite metagranitoids (59 to 70 wt. \%). The augen metagranitoids are classified as granites and granodiorites in the normative $\mathrm{Ab}-\mathrm{An}-\mathrm{Or}$ diagram (O'Connor 1965) and all of the analysed samples of migmatitic augen gneisses correspond to granite (Fig. 8). Slightly different results are obtained in the $\mathrm{SiO}_{2}$ vs. $\mathrm{K}_{2} \mathrm{O}+\mathrm{Na}_{2} \mathrm{O}$ diagram (Middlemost 1994) with augen metagranitoids plotting in the fields of granite, granodiorite, quartz monzodiorite and tonalite. The migmatitic augen gneisses are classified as granite and granodiorite in a good agreement with the Ab-An-Or plot (Fig. 8).

All of the studied samples show relatively high $\mathrm{K}_{2} \mathrm{O}$ abundance in the $\mathrm{SiO}_{2}-\mathrm{K}_{2} \mathrm{O}$ diagram (Peccerillo and Taylor 1976; Fig. 9); the bulk of the augen migmatitic gneiss samples fall within the high-K calc-alkaline series field. Even though the augen metagranitoids show a relatively wide data dispersion, most of the samples plot in the high-K calc-alkaline field.

The samples of both studied groups are metaluminous to peraluminous $[\mathrm{A} / \mathrm{CNK}=0.9-1.4$ where $\mathrm{A} / \mathrm{CNK}=$ molar $\left.\mathrm{Al}_{2} \mathrm{O}_{3} /\left(\mathrm{CaO}+\mathrm{Na}_{2} \mathrm{O}+\mathrm{K}_{2} \mathrm{O}\right)\right]$; migmatitic augen gneisses with sillimanite are strongly peraluminous $(\mathrm{A} / \mathrm{CNK}=1.9)$.

In general, augen metagranitoids and migmatitic augen gneisses exhibit similar chondrite-normalized REE patterns (Boynton, 1984), characterized by enrichment in light rare earth elements $\left(\mathrm{La}_{\mathrm{N}} / \mathrm{Yb}_{\mathrm{N}}=3.5-14.7\right)$ and relative depletion in heavy rare earth elements (HREE) (Fig. 10a-b). Migmatitic augen gneisses show usually more pronounced negative $\mathrm{Eu}$ anomalies $\left(\mathrm{Eu} / \mathrm{Eu}^{*}=0.28\right.$ 0.71 ) and slightly higher content of HREE than augen metagranitoids $\left(\mathrm{Eu} / \mathrm{Eu}^{*}=0.54-0.93\right)$. Total REE abundance in both rock groups is similar (129-232 ppm).

In all of the studied samples, $\mathrm{TiO}_{2}, \mathrm{Al}_{2} \mathrm{O}_{3}$, total $\mathrm{FeO}$, $\mathrm{MgO}, \mathrm{P}_{2} \mathrm{O}_{5}, \mathrm{~V}, \mathrm{Sr}$, and Co contents decrease with increasing $\mathrm{SiO}_{2}$ (Fig. 9). Based on the chemical composition, augen metagranitoids are subdivided into two subgroups. Chemical composition of the first subgroup agrees well with chemical composition of the tonalite group from the Budislav Pluton. The augen metagranitoids have higher contents of $\mathrm{MgO}$ (2.2-3.5 wt. \%), $\mathrm{CaO}$ (3.4-5.4 wt. \%), $\mathrm{Sr}$ (199-441 ppm), Ba (973-1110 ppm) and higher $\mathrm{Ba} / \mathrm{Rb}$ ratios (8-9). In contrast, the chemical composition of the second subgroup of augen metagranitoids is significantly different (Fig. 8). Characteristic is lower content of $\mathrm{CaO}$ (1.0-2.0 wt. \%), $\mathrm{MgO}$ (1.1-2.6 wt. \%; Fig. 9), and lower $\mathrm{Ba} / \mathrm{Rb}$ ratios (4-5).

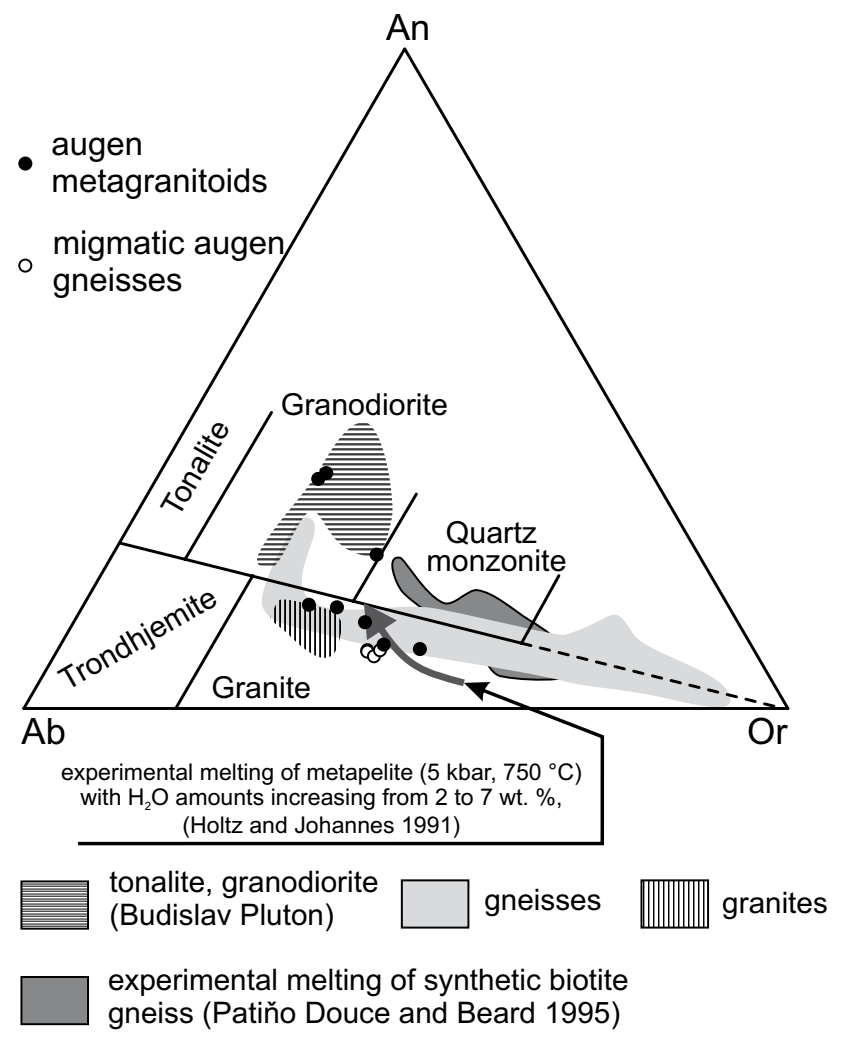

Fig. 8 Ternary An-Ab-Or diagram used for classification of siliceous igneous rocks (O’Connor 1965), based on CIPW norm.

The migmatitic augen gneisses contain less $\mathrm{Al}_{2} \mathrm{O}_{3}$, $\mathrm{FeO}_{\mathrm{t}}, \mathrm{TiO}_{2}, \mathrm{MgO}$ and $\mathrm{V}$ and have higher $\mathrm{K}_{2} \mathrm{O}, \mathrm{SiO}_{2}$ and $\mathrm{Y} / \mathrm{Zr}$ compared with average augen metagranitoids (Fig. 11a-b, d).

\section{Discussion}

The augen gneisses, conspicuous rocks with suboval feldspar "pearls" set in dark grey fine- to medium-grained matrix, dominate in the lower subunit of the PU most of all along the contact with the underlying Svratka Unit. Even though they form a homogenous body, detailed petrological and petrochemical studies distinguished two groups of augen gneiss: augen metagranitoids and augen migmatitic gneisses. Development of the two groups is related to Variscan magmatic event accompanying partial melting and subsequent deformation in the PU. The main magmatic phase in the PU was dated by Vondrovic and Verner (2008) at $350 \mathrm{Ma}$ and it coincide with the early stages of exhumation processes in the eastern Bohemian Massif (Schulmann et al. 2005). This tectonic process ultimately caused the exhumation of deeper crustal segments - highly metamorphosed granulitic rocks - dated by Schulmann et al. (2005) at 340 Ma. Moreover, it triggered partial melting in the hosting medium- to highgrade metamorphic complexes. 

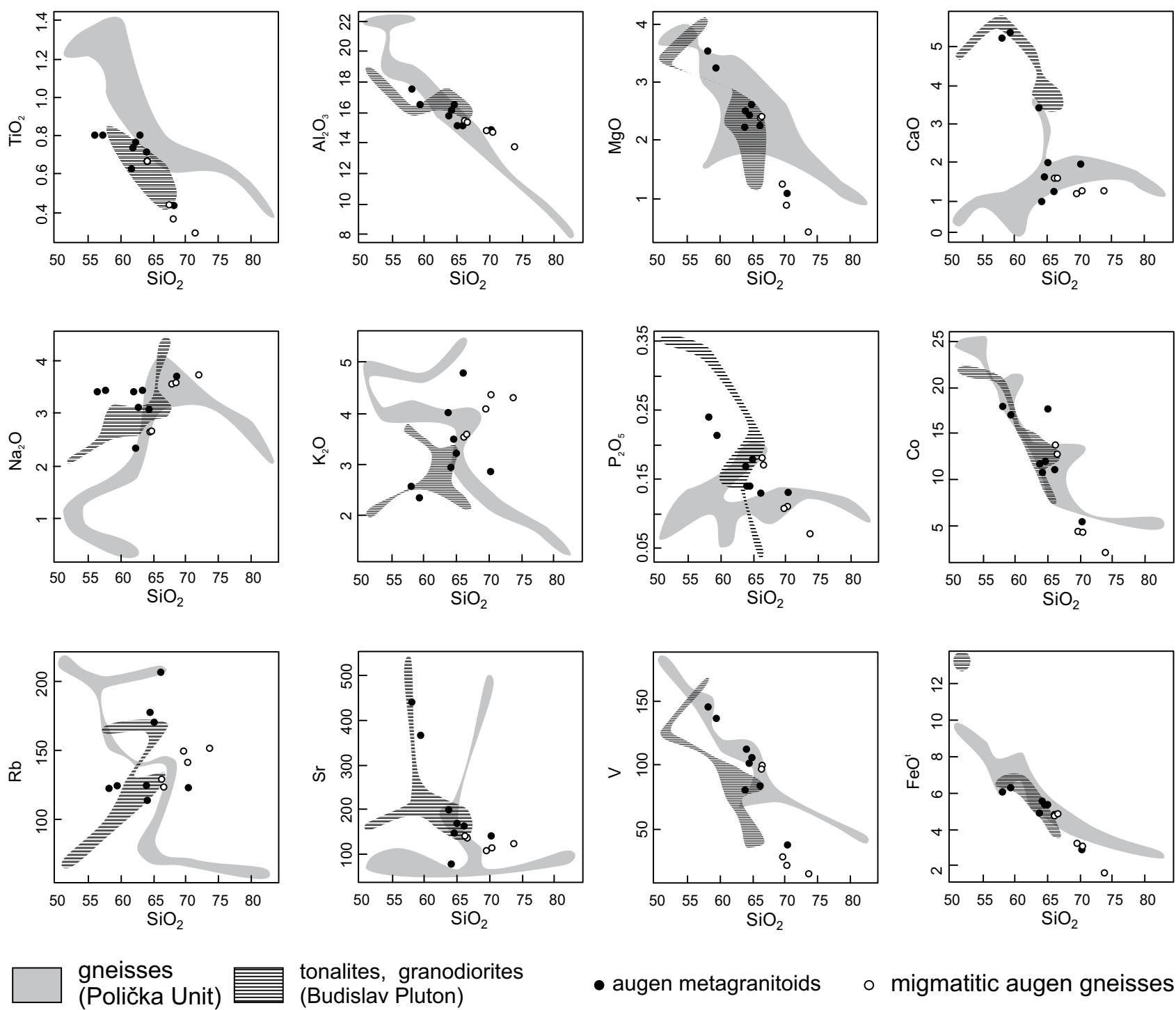

tonalites, granodiorites (Budislav Pluton)

- augen metagranitoids

○ migmatitic augen gneisses

Fig. 9 Binary variation diagrams of silica with selected major and trace elements in the augen gneisses, gneisses and rocks of the Budislav Pluton (Polička Unit).

\subsection{Magmatic events}

The lower and middle units of the PU are commonly intruded by the Variscan intrusive bodies (Buriánek et al. 2003; Vondrovic and Verner 2008) among which the Miřetín and Budislav plutons are the largest. The Miřetín Pluton crystallized at a pressure of about 3-4 kbar (Hanžl ed. 2008) in the NW part of the lower subunit of PU. Similar igneous rocks and migmatites in the $\mathrm{S}$ part crystallized at the pressure of $c .7 \mathrm{kbar}$ (Fig. 7). The age of intrusion is estimated at $348 \pm 7 \mathrm{Ma}$ by U-Pb zircon method (Vondrovic and Verner 2008). Medium- to coarse-grained, often porphyritic granodiorites, tonalites and diorites were subsequently deformed into high- and low-temperature solid-state fabrics (Vondrovic and Verner 2008).
Emplacement of the Budislav Pluton in the middle subunit of the PU has been dated at $350 \pm 5$ Ma by the $\mathrm{U}-\mathrm{Pb}$ zircon method (Vondrovic and Verner 2008). It was contemporaneous with the $\mathrm{M}_{2}$ metamorphic peak in the PU, P-T conditions of which were estimated at 600 ${ }^{\circ} \mathrm{C}$ and 6 kbar. Besides larger plutonic bodies, numerous sills and dykes of granites to granodiorites of thickness reaching centimetres to first metres crosscut migmatitized metasediments of the lower subunit of the PU along the contact with the Svratka Unit. The prevailing microstructural features indicate progressive cooling during the deformation in igneous dykes and migmatites.

The emplaced magma represented a major heat and fluid source. The surrounding metapelites were thus affected not only by thermal metamorphism, but also by a fluid ingress. Fluid overpressure triggered a water-satu- 

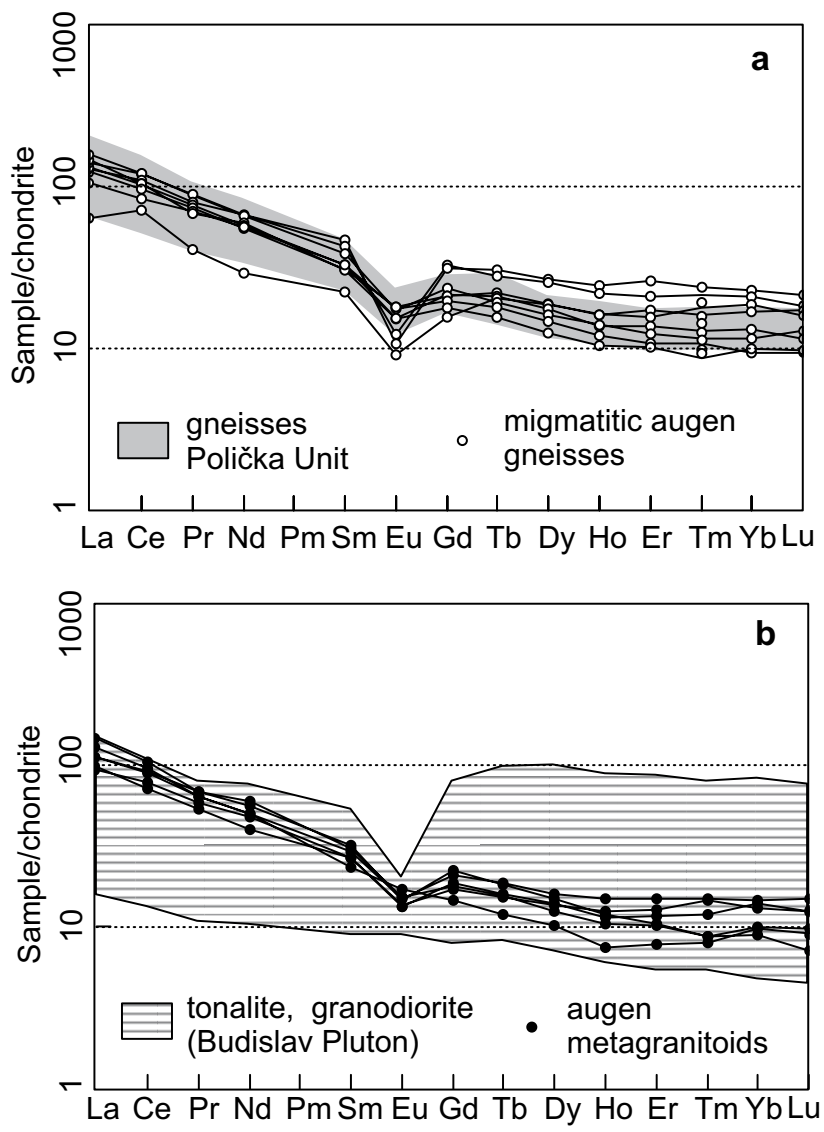

Fig. 10 Chondrite-normalized REE patterns (Boynton 1984) for augen gneisses, gneisses (a) and rocks of the Budislav Pluton (b).

rated melting, producing small patches of low-K granite melt as a source for plagioclase crystallization.

\subsection{Augen metagranitoids}

In the northern part of the PU is locally preserved a complete transition from the mesoscopically nearly undeformed porphyritic granodiorites of the Miřetín Pluton with euhedral plagioclase phenocrysts to the strongly foliated and recrystallized augen orthogneisses. All samples show substantial solid-stage deformation. Similar mylonitic augen microstructures were produced experimentally by dislocation creep accompanied by grain size reduction due to a rather high-T recrystallization at about $650{ }^{\circ} \mathrm{C}$ (Tullis and Yund 1987).

The prevailing type of augen gneiss developed from medium-grained rocks with granitic chemical compositions. These rocks originated by partial melting of metapelites. This is suggested by the presence of abundant biotite and sillimanite-rich enclaves of restitic character, composition of plagioclases (Fig. 4), as well as chemistry and zoning of garnets (Fig. 6). Plagioclase composition is usually comparable with that in the rocks of tonalite suite (Fig. 4). However some samples contain plagioclase with a higher $\mathrm{P}_{2} \mathrm{O}_{5}$ content. The phosphorus content in the feldspar from granitic rocks often increases with peraluminosity expressed by the $\mathrm{A} / \mathrm{CNK}$ parameter (Broska et al. 2004). Relatively high phosphorus contents have been determined in alkali feldspars from many peraluminous granites worldwide (e.g., London 1992) and also in the metapelites of the PU (Fig. 4). Whole-rock chemical composition of metagranitoids indicates mixing between tonalite and crustal melts derived by partial melting of metapelites in the PU (Figs 8-9, 11). This process occurred at deeper crustal levels and the hybrid magmas migrated to the zone of deformation.

\subsection{Migmatitic augen gneisses}

Migmatitic augen gneisses originated by partial melting of a metasedimentary protolith in the lower subunit of the PU. Partial melting was probably caused by advected heat from the tonalite group rocks (Miřetín and Budislav plutons). However the migmatitic augen gneisses have not been dated yet. Residual fluids expelled from granitoids seem to have pervasively infiltrated the mica-rich metasediments and enhanced the melting. In the finegrained biotite gneisses, the melt appears only rarely in discrete accumulation. It usually forms several $\mathrm{cm}$ thick veins parallel with foliation or it is entirely missing.

Close relation between magmatic activity and partial melting is indicated by the chemical composition of rocks. The REE patterns of the migmatitic augen gneisses $\left(\right.$ median $\left.\mathrm{Eu}_{\mathrm{N}} / \mathrm{Yb}_{\mathrm{N}}=0.85\right)$ are very similar to those of the gneisses (Fig. 10a), except for a slight enrichment in HREE (median $\mathrm{Eu}_{\mathrm{N}} / \mathrm{Yb}_{\mathrm{N}}=1.50$ ). This is in a good agreement with the differences between gneisses (median $\left.\mathrm{Eu}_{\mathrm{N}} / \mathrm{Yb}_{\mathrm{N}}=1.06\right)$ and tonalite group rocks (median $\mathrm{Eu}_{\mathrm{N}} /$ $\mathrm{Yb}_{\mathrm{N}}=1.36$ ) in the PU (Fig. 10a-b). The REE patterns in migmatitic augen gneisses are very similar to those of the augen metagranitoids with slightly higher HREE contents, which can be explained by an enrichment of HREE-rich accessory minerals like zircon and xenotime in the biotite-rich restite. The high Y/Zr ratio (Fig. 11b), indicates a significant contribution of xenotime to the HREE budget. Zirconium decreases with increasing $\mathrm{SiO}_{2}$ content in rocks. This chemical correlation may reflect the increasing leucosome content in the migmatitic augen gneisses. During prograde metamorphism of metapelites, The HREE are preferentially concentrated in xenotime (Pyle and Spear 1999). Dissolution of xenotime during partial melting would produce melts with higher $\mathrm{Y}$ and HREE contents. The trace-element composition of garnet is controlled by partitioning with melt (Pyle and Spear 1999). Garnet is minor but typical constituent of migmatitic augen gneisses and it displays slightly higher $\mathrm{Y}_{2} \mathrm{O}_{3}$ (0.03-0.19 wt. \%) contents than the garnet from other 

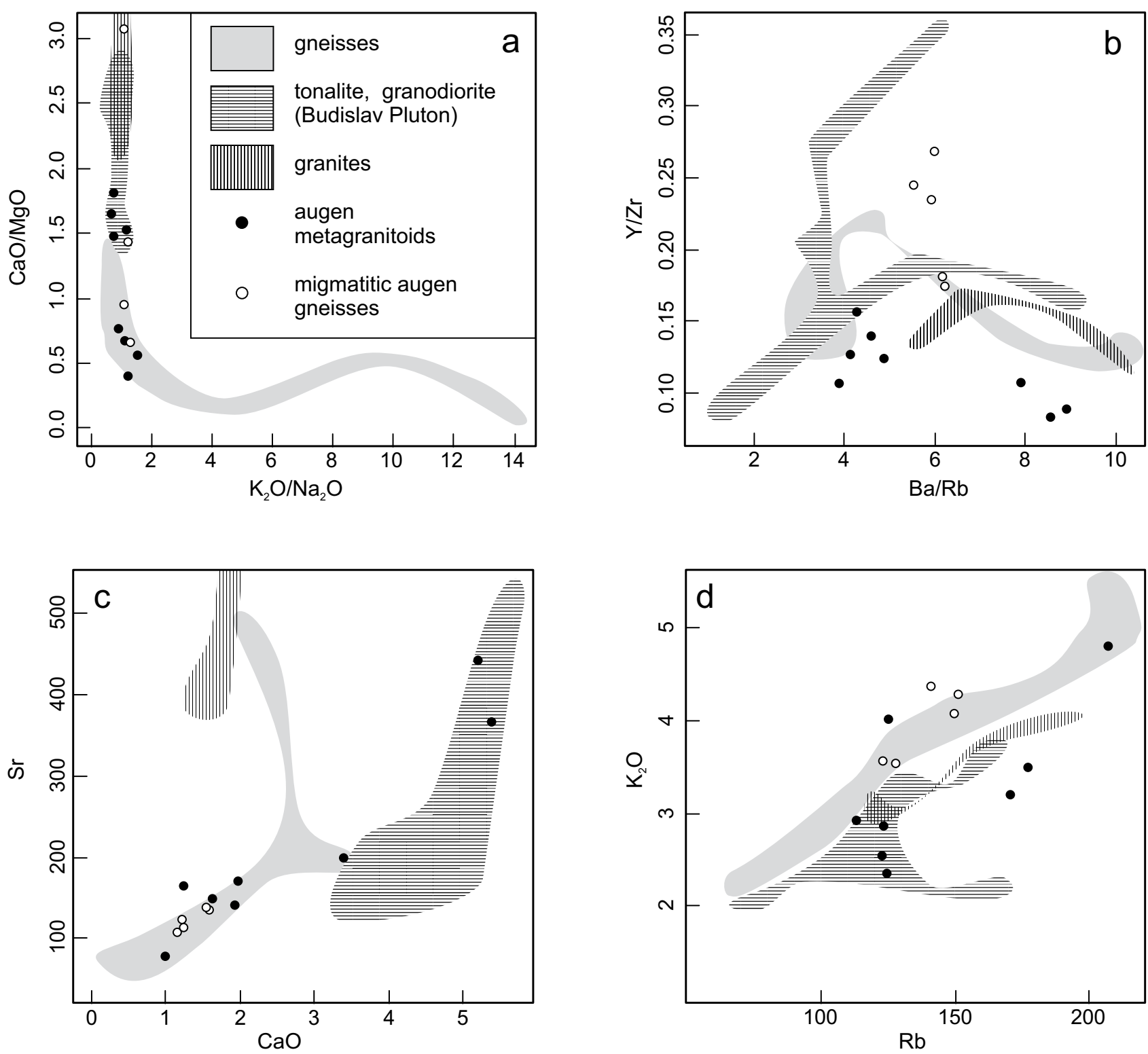

Fig. 11 The whole-rock $\mathrm{K}_{2} \mathrm{O} / \mathrm{Na}_{2} \mathrm{O}-\mathrm{CaO} / \mathrm{MgO}(\mathbf{a}), \mathrm{Ba} / \mathrm{Rb}-\mathrm{Y} / \mathrm{Zr}(\mathbf{b}), \mathrm{CaO}-\mathrm{Sr}(\mathbf{c})$ and $\mathrm{Rb}-\mathrm{K}_{2} \mathrm{O}(\mathbf{d})$ diagrams for augen gneisses, gneisses and the rocks of Budislav Pluton.

gneisses of the PU (0.00-0.16 wt. \%). Tonalite group rocks with higher $\mathrm{Y}$ and HREE contents contain significant amount (8-15 vol. \%) of garnet (Fig. 10b).

The geochemistry of $\mathrm{Rb}, \mathrm{Sr}$ and $\mathrm{Ba}$ (Fig. 11) is strongly influenced by the balance of feldspars and micas in the source and the mechanism of melting. For the typical muscovite-rich pelite, dehydratation melting leads to a liquid enriched in $\mathrm{Rb}$ and depleted in $\mathrm{Sr}$ and $\mathrm{Ba}$ (Harris and Inger 1992). In contrast to melts produced through water-saturated melting, it will have low $\mathrm{Sr} / \mathrm{Ba}$ and $\mathrm{Rb} /$ $\mathrm{Sr}$ ratios. The $\mathrm{Rb} / \mathrm{Sr}$ ratios are also reduced under disequilibrium melting conditions irrespective of the water activity during melting (Harris et al. 1993). The low Rb/ Sr ratios (0.9-1.4) and high Ba contents (763-887 ppm) in the migmatitic augen gneisses indicate water-present melting (Harris et al. 1993). The interpretation of the $\mathrm{K}_{2} \mathrm{O}-\mathrm{Rb}$ and $\mathrm{Sr}-\mathrm{CaO}$ plots (Fig. 11c and 11d) indicates that migmatitic augen gneisses may have formed by melting of metasedimentary sequence of the PU. The position of migmatitic augen gneisses in the normative $\mathrm{An}-\mathrm{Ab}-\mathrm{Or}$ diagram (Fig. 8) suggests an anatexis of metapelites rather than of greywackes (Patiño Douce and Beard 1995). With increase in $\mathrm{H}_{2} \mathrm{O}$ at constant P-T conditions the content of alkali feldspar component in the melt decreases (Holtz and Johannes 1991). The elements concentrated in mafic minerals (e.g. Mg, Fe, Ti) show good linear correlations with $\mathrm{SiO}_{2}$ from augen metagranitoids to the migmatitic augen gneisses (Fig. 9). Much of the 
major- and trace-element variability can be explained by mixing of tonalitic magma and melt generated by anatexis of the metasediments. Microtextural features indicate retrograde breakdown of $\mathrm{K}$-feldspar in migmatites according the reaction: $\mathrm{Kfs}+\mathrm{Sil}+\mathrm{H}_{2} \mathrm{O}=\mathrm{Ms}+$ Qtz.

The migmatitic gneisses contain augen composed of plagioclase grains or quartz-plagioclase aggregates, which are interpreted as a result of combination of in situ partial melting and deformation.

The observed geochemical and mineralogical characteristics and the spatial relations of migmatitic augen gneisses (Fig. 12a-b) and augen metagranitoid sheets (Fig. 12c) lead to the interpretation of the migmatitic augen gneisses as a result of external fluids infiltration.
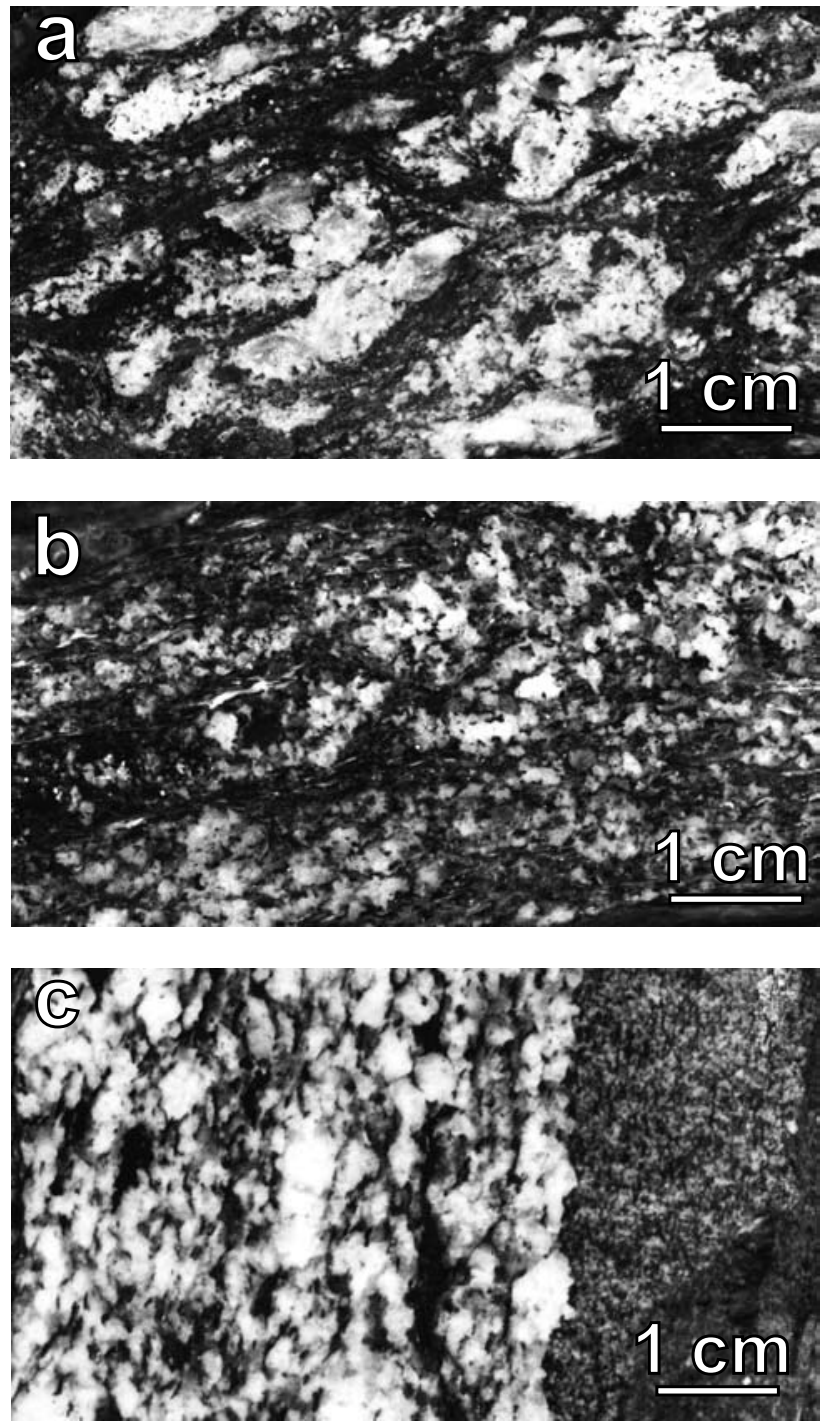

Fig. 12 Typical textures in slightly deformed rocks parental to the augen gneisses (transitions between textures of migmatite and granodiorite). a - Migmatitic augen gneiss with visible S-C fabric, (sample 68, Bystré). $\mathbf{b}$ - Migmatitic augen gneiss with sillimanite and biotite-rich enclaves, Ubušín (sample 323). c - Contact between fine-grained biotite gneiss and $7 \mathrm{~cm}$ thick metagranitoid dyke, Jedlová (sample 131).
These were most likely residual fluids from granitoid magmas that infiltrated the metasedimentary protolith during regional metamorphism $\mathrm{M}_{2}$.

Loaded solid-state deformation during the final stages of the Svratka and the Polička units convergence and later normal faulting along the SE margin of Hlinsko Unit obscured the differences between the granitic and migmatitized gneiss-derived augen gneisses.

\section{Conclusions}

Augen gneisses of the Polička Unit appear as a homogenous rock group at the first sight. However, within this group exist significant differences in textural and petrochemical characteristics. Two main subgroups were distinguished: augen metagranitoids and migmatitic augen gneisses. Both represent cogenetic products of the same processes operative along the contact of the Svratka and the Polička units.

During the first stage tonalitic to granodioritic melt intruded a metapelite protolith of the Polička Unit in the form of sills and dykes. Residual fluids released during this magmatic event pervasively infiltrated the metasedimentary host rocks and caused a fluid-present partial melting. The P-T conditions of partial melting during the metamorphic event $\mathrm{M}_{2}$ were estimated at $\sim 750-800$ ${ }^{\circ} \mathrm{C}$ and $7 \mathrm{kbar}$. The fluid-present partial melting resulted in the origin of opthalmitic and stromatitic migmatites around granitoid intrusions.

Augen metagranitoids and migmatitic augen gneisses were both affected by the same ductile shearing. Microstructural characteristics and mineral fabrics provide evidence for high-temperature deformation of migmatites and tonalites in subsolidus conditions.

Emplacement of granodiorite to tonalite bodies and their sub-solidus deformations were associated with ductile shear zone, representing a metamorphic event $\left(\mathrm{M}_{2}\right)$ and exhumation of high-grade complexes, including granulites. Kinematic features in augen gneisses indicate displacement of the Polička over the Svratka Unit. These structures can be interpreted as a result of crustal-scale folding with NW-directed overthrusting of the Bohemicum over the Svratka Unit. Small occurrences of mainly igneous rocks of the PU within the Moldanubian Zone and along the northern rim of the Svratka Unit indicate a larger areal extent of the Bohemicum nappe above the Svratka Unit and Moldanubian Zone than supposed previously.

Acknowledgements The authors wish to thank T. ObercDziedzic and M. Putiš for critical reviews of the manuscript. The work was conducted with the financial aid of the Czech Ministry of Environment, Project No. 6352: 
'Correlation of Lithologically Contrasting Rocks From the Crystalline Units on the NE boundary of Moldanubicum' and Grant Agency of the Charles University, Project No. 81909: 'The Emplacement Mechanism of Individual Plutons in Northern Part of the Moldanubia; Implications for Tectonic Evolution of the Eastern Margin of the Bohemian Massif'. We are grateful to R. Melichar for his comments and suggestions during the manuscript preparation.

Electronic supplementary material. The Tab. 6, as well as GPS coordinates of the studied samples, are available online at the Journal web site www.jgeosci.org.

\section{References}

ANDERSon JL, SMith DR (1995) The effects of temperature and $\mathrm{fO}_{2}$ on the Al-in-hornblende barometer. Amer Miner 80: 549-559

BOullier AM, Bouchez JL (1978) Le quartz en rubans dans les mylonites. Bull Soc Geol France 7: 253-262

BOYNTON WV (1984) Cosmochemistry of the rare earth elements: meteorite studies. In: Henderson PE (ed) Rare Earth Element Geochemistry. Developments in Geochemistry. Elsevier, Amsterdam, pp 63-114

Broska I, Williams CT, Uher P, Konecný P, Leichmann J (2004) The geochemistry of phosphorus in different granite suites of the Western Carpathians, Slovakia: the role of apatite and P-bearing feldspar. Chem Geol 205: 1-15

BuriáNeK D, NĚMeČKovÁ M, HANŽL P (2003) Petrology and geochemistry of the plutonic rocks in the Polička and Zábřeh crystalline units (NE Bohemian Massif). Bull Geosci 78: 9-22

Buriánek D (ed), Břízová E, Čech S, Čurda J, Fürych V, HaNŽL P, Kirchner K, Lysenko V, RoštínsKÝ P, RÝdA K, Skácelová D, Skácelová Z, Verner K, Vít J (2009) Basic geological map ČR 1:25 000 with explanations, 24-112 Jedlová. Czech Geological Survey, Prague, pp 1-76 (in Czech)

Buriánková K, HanžL P, Buriánek D (2006) Occurrence of rocks of Polička Crystalline Unit in Moldanubian assemblage near Stržanov. In: KRMíčEK L (ed) Moravskoslezské Paleozoikum 2006, Book of Abstracts. Masaryk University, Brno, pp 8 (in Czech)

Čéh S (ed), BŘizová E, Buriánek D, Čurda J, Fürych V, KirChNER K, Lysenko V, MrNKová J, RoŠTínsKÝ P, RÝdA K, SkÁcelová Z, Vít J (2009) Basic geological map ČR 1:25 000 with explanations, 14-334 Polička. Czech Geological Survey, Prague, pp 1-65 (in Czech)

GuidotTi CV (1984) Micas in metamorphic rocks. In: BAILEY SW (ed) Micas. Mineral Soc Amer Rev Mineral Geochem 13: pp 357-467

HanžL P (ed), Buriánek D, Čurda J, Fürich V, HrdličKová K, Kirchner K, Lysenko V, Mrnková J, Otava J, Per-
TOldová J, RoštínskÝ P, RÝda K, SKÁcelová Z, Vít J, ZELENKA P (2008) Basic geological map ČR 1:25 000 with explanations, 14-333 Svratka. Czech Geological Survey, Prague, pp 1-72 (in Czech)

Harris N, Inger S, Massey J (1993) The role of fluids in the formation of the High Himalayan leucogranites. In: Treloar PJT, Searle MP (eds) Himalayan Tectonics. Geol Soc London Spec Publ 74: 391-400

HARRIS N, INGER S (1992) Trace element modelling of pelite derived granites. Contrib Mineral Petrol 110: 46-56

Holdaway MJ, Mukhopadhyay B (1993) A re-evaluation of the stability relations of andalusite: thermochemical data and phase diagram for the aluminum silicates. Amer Miner 78: 298-315

Holland TJB, Blundy, JD (1994) Non-ideal interactions in calcic amphiboles and their bearing on amphiboleplagioclase thermometry. Contrib Mineral Petrol 116: 433-447

Holland TJB, Powell R (1998) An internally consistent thermodynamic data set for phases of petrological interest. J Metamorph Geol 16: 309-343

Holtz F, Johannes W (1991) Genesis of peraluminous granites I. experimental investigation of melt compositions at 3 and $5 \mathrm{~kb}$ and various $\mathrm{H}_{2} \mathrm{O}$ activities. J Petrol 32: $935-958$

HrdličKová K (ed), BŘízová E, Fürych V, Hanžl P, Kadlecová R, Kirchner K, Lysenko V, Lhotský P, Mrnková J, Pertoldová J, Roštínský P, Skácelová D, Skácelová Z, VíT J (2008) Basic geological map ČR 1:25 000 with explanations, 23-224 Žd'ár nad Sázavou. Czech Geological Survey, Prague, pp 1- 60 (in Czech)

Hutton DHW, Reavy RJ (1992) Strike-slip tectonics and granite petrogenesis. Tectonics 11: 960-967

JANOUŠEK V, FARROW CM, ERBAN V (2006) Interpretation of whole-rock geochemical data in igneous geochemistry: introducing Geochemical Data Toolkit (GCDkit). J Petrol 47: 1255-1259

KRETZ R (1983) Symbols for rock-forming minerals. Amer Miner 68: 277-279

Leake BE, Wooley AR, Arps CES, Birch WD, Gilbert MC, Grice JD, Hawthorne FC, Kato A, Kisch HJ, KrivoviCHEV VG (1997) Nomenclature of amphiboles: report of the Subcommittee on Amphiboles of the International Mineralogical Association Commission on New Minerals and Mineral Names. Canad Mineral 35: 219-237

London D (1992) Phosphorus in S-type magmas: the $\mathrm{P}_{2} \mathrm{O}_{5}$ content of feldspars from peraluminous granites, pegmatites and rhyolites. Amer Miner 77: 126-145

Melichar R (1993) Structural analysis of the Polička and Svratka crystalline units. Unpublished $\mathrm{PhD}$ thesis Charles University, Prague, pp 1-50 (in Czech)

Melichar R (ed), Buriánek D, Břizová E, Buriánková K, Čurda J, FÜrych V, HaNžL P, KirChNER K, LysenKo V, Mrnková J, RoštínsKÝ P, RÝdA K, Skácelová Z, Vít J 
(2008) Basic geological map ČR 1:25 000 with explanations, 24-111 Sněžné. Czech Geological Survey, Prague, pp 1-58, (in Czech)

Melichar R, HanžL P (1997) Lithotectonic correlation of the Polička and Zábřreh crystalline units. J Czech Geol Soc 42: 64

MeHnert WD (1968) Migmatites and Origin of Granitic Rocks. Elsevier, Amsterdam, pp 1-393

Middlemost EAK (1994) Naming materials in the magma/ igneous rock system. Earth Sci Rev 37: 215-224

MísAR̆ Z (1961) Geological position and development of the leucocratic granite gneisses in surroundings of Vír. Sborník Ústř úst geol 28: 31-52 (in Czech)

Mísař Z, Dudek A, Havlena V, Weiss J (1983) Geology of ČSSR I, Bohemian Massif. SPN, Prague, pp 1-333 (in Czech)

Mrázová Š (ed), Břizová E, Buriánek D, Fürych V, KaDLECovÁ R, KirChNER K, LySENKo V, OtAVA J, RAmbousek P, Roštínský P, SkÁcelová D, SkÁcelová Z, Vít J, ZeLENKA P (2008) Basic geological map ČR 1:25 000 with explanations, 13-444 Hlinsko. Czech Geological Survey, Prague, pp 1-68 (in Czech)

O'CONNOR JT (1965) A classification for quartz-rich igneous rocks based on feldspar ratios. US Geological Survey Professional Paper B525, pp 79-84

Patiño Douce AE, Beard JS (1995) Dehydration-melting of biotite gneiss and quartz amphibolite from 3 to $15 \mathrm{kbar}$. J Petrol 36: 707-738

Peccerillo A, Taylor SR (1976) Geochemistry of Eocene calc-alkaline volcanic rocks from the Kastamonu area, Northern Turkey. Contrib Mineral Petrol 58: 63-81

Pinarelli L, Bergomi MA, Boriani A, Giobbi E (2008) Pre-metamorphic melt infiltration in metasediments: geochemical, isotopic ( $\mathrm{Sr}, \mathrm{Nd}$, and $\mathrm{Pb})$, and field evidence from Serie dei Laghi (Southern Alps, Italy). Mineral Petrol 93: 213-242

Pitra P, Guiard M (1996) Probable anticlockwise evolution in extending crust: Hlinsko region, Bohemian Massif. J Metamorph Geol 14: 49-60

Powell R, Holland TJB (1988) An internally consistent dataset with uncertainties and correlations; 3, Applications to geobarometry, worked examples and a computer program. J Metamorph Geol 6: 173-204

PyLE JM, Spear FS (1999) Yttrium zoning in garnet: coupling of major and accessory phases during metamorphic reactions. Geol Mat Res 1: 1-49
Rejchrt M, Břizová E, Fürych V, Hanžl P, Hradecká L, HrdičKová K, Kadlecová R, Kirchner K, Lysenko V, Mlčoch B, Nahodilová R, Novák M, Otava J, Pertoldová J, Rambousek P, Roštínský P, Rudolský J, Skácelová D, Skácelová Z, Týcová P, Vít J, Žáč́ová E (2009) Basic geological map ČR $1: 25000$ with explanations, 23-222 Krucemburk. Czech Geological Survey, Prague, pp 1-58 (in Czech)

Rosiwal A. (1895) Aus den kristallinischen Gebiete des Oberlaufes der Schwarzawa V. Verh Geol Reichsanst (Wien) 8: 231-242

Schulmann K, Kröner A, Hegner E, Wendt I, Konopásek J, LEXA O, ŠTíPSKÁ P (2005) Chronological constraints on the pre-orogenic history, burial and exhumation of deep seated rocks along the eastern margin of the Variscan orogen, Bohemian Massif, Czech Republic. Amer J Sci 305: 407-448

StÁrkovÁ I, MaceK J (1994) Geological map ČR 1:50 000, 24-13 Bystřice nad Pernštejnem. Czech Geological Survey, Prague

Štoudová S, Schulmann K, Konopásek J (1999) A contrast between metamorphic and structural evolution of the Vír granulite and surrounding rocks of the Polička Crystalline Unit. Geolines 8: 64-65

Tajčmanová L, Verner K, Pertoldová J. (2005) Correlation of structural and metamorphic evolution of metamorphic rocks from the Svratka and Polička crystalline complexes. Geolines 19: 112

TulLIS J, Yund RA (1987) Transition from cataclastic flow to dislocation creep of feldspar: mechanisms and microstructures. Geology 15: 606-609

VAssallo JJ, Vernon RH (2000) Origin of megacrystic felsic gneisses at Broken Hill. Austr J Earth Sci 47: 733-748

Vernon RH., Paterson SR (2002) Igneous origin of Kfeldspar megacrysts in deformed granites of the Papoose Flat Pluton, California, USA. Electronic Geosciences 7: 31-39

VondRovic L, Verner K (2008) The record of structural evolution and $\mathrm{U}-\mathrm{Pb}$ zircon dating of the tonalite intrusions (Polička Crystalline Unit, Bohemian Massif). In: Poblet J, Medina MG, Pedreira D, Fernández Cl (eds) International Meeting of Young Researches in Structural Geology and Tectonics, Programme and Extended Abstracts, University of Oviedo, Spain, pp 369-373

White S. (1975) Tectonic deformation and recrystallization of oligoclase. Contrib Mineral Petrol 50: 287-304 